\title{
Higgs critical exponents and conformal bootstrap in four dimensions
}

\author{
Oleg Antipin, ${ }^{a}$ Esben Mølgaard $^{b}$ and Francesco Sannino ${ }^{b}$ \\ ${ }^{a}$ INFN, Sezione di Firenze, \\ Via G. Sansone 1, I-50019 Sesto Fiorentino, Italy \\ ${ }^{b} C P^{3}$-Origins 86 the Danish Institute for Advanced Study Danish IAS, \\ University of Southern Denmark, \\ Campusvej 55, DK-5230 Odense M, Denmark \\ E-mail: antipin@fi.infn.it, molgaard@cp3.dias.sdu.dk, \\ sannino@cp3.dias.sdu.dk
}

ABSTRACT: We investigate relevant properties of composite operators emerging in nonsupersymmetric, four-dimensional gauge-Yukawa theories with interacting conformal fixed points within a precise framework. The theories investigated in this work are structurally similar to the standard model of particle interactions, but differ by developing perturbative interacting fixed points. We investigate the physical properties of the singlet and the adjoint composite operators quadratic in the Higgs field, and discover, via a direct computation, that the singlet anomalous dimension is substantially larger than the adjoint one. The numerical bootstrap results are, when possible, compared to our precise findings associated to the four dimensional conformal field theoretical results. To accomplish this, it was necessary to calculate explicitly the crossing symmetry relations for the global symmetry group $\mathrm{SU}(N) \times \mathrm{SU}(N)$.

Keywords: Nonperturbative Effects, Conformal and W Symmetry, 1/N Expansion, Renormalization Group

ARXIV EPRINT: 1406.6166 


\section{Contents}

1 Introduction 1

2 Conformal bootstrap review 2

3 Conformal bootstrap sum rules in CFT with $\mathrm{SU}\left(N_{f}\right)_{L} \times \mathrm{SU}\left(N_{f}\right)_{R}$ global symmetry $\quad 6$

4 A four-dimensional calculable Gauge-Yukawa CFT $\quad 8$

$\begin{array}{llr}4.1 \text { Beta functions and Weyl consistency conditions } & 9\end{array}$

$\begin{array}{lll}4.2 & \text { Higgs anomalous dimensions } & 11\end{array}$

$\begin{array}{lll}4.3 & \text { Bootstrap in the Veneziano limit } & 12\end{array}$

$\begin{array}{llr}4.4 & \text { Physical results } & 16\end{array}$

$\begin{array}{ll}\text { 4.4.1 The } \ell=0 \text { case } & 16\end{array}$

$\begin{array}{ll}\text { 4.4.2 The } \ell=1 \text { case } & 17\end{array}$

$\begin{array}{llr}5 & \text { Conclusions } & 17\end{array}$

$\begin{array}{lr}\text { A The u-channel sum rule } & 19\end{array}$

B Complete beta functions and anomalous dimensions beyond the

$\begin{array}{ll}\text { Veneziano limit } & 20\end{array}$

\section{Introduction}

The discovery of a Higgs particle at the Large Hadron Collider is a major leap forward towards the construction of a more complete theory of nature. If the discovered particle is the standard model Higgs, it is imperative to understand the gauge dynamics of nonsupersymmetric four-dimensional gauge-Yukawa theories.

Among all possible quantum field theories, the ones developing quantum conformal fixed points have a central role [1, 2]. Quantum chromodynamics is a time-honored example [3, 4], where the celebrated property of asymptotic freedom comes from a noninteracting ultraviolet fixed point $[3,4]$. One can also imagine the existence of ultraviolet fixed points that are interacting, and this scenario is referred to as asymptotic safety [5].

Recently, phenomenological ultraviolet conformal extensions of the standard model with and without gravity have received much attention [6-21]. More generally, model building requiring scale invariance both in particle physics and cosmology [22-46] is an active area of research. Furthermore, following Weinberg [5], even quantum aspects of gravity can be addressed in an asymptotic safety scenario [47-53]. However, in four dimensions, asymptotic safety has only recently [54] been guaranteed to occur in calculable 
nonsupersymmetric gauge-Yukawa theories. Last but not least, perturbative and nonperturbative infrared interacting fixed points are very interesting, both theoretically and phenomenologically [55-81]. For infrared non-perturbative fixed points in gauge theories, lattice computations are making remarkable progress [82-99].

In this work, we therefore wish to press forward and investigate explicit conformal properties of nonsupersymmetric gauge-Yukawa theories. We are particularly interested in the properties associated with enforcing crossing symmetry on four-point correlation functions. The microscopic theories investigated here are $\mathrm{SU}\left(N_{c}\right)$ gauge theories featuring $N_{f}$ Dirac fermions transforming according to the fundamental representation of the gauge group, $\ell$ adjoint Weyl fermions, and $N_{f}^{2}$ complex scalars, encapsulated in the Higgs matrix $H$. The scalars are coupled to the fermion and gauge sectors via Yukawa interactions. The existence of Banks-Zaks (BZ) [100] interacting fixed points in such a model has been established in [75, 101-104]. Furthermore, in [54] the reader will find an in depth study of the asymptotic safety scenario and crucial properties which are guaranteed to exist for some of these theories. In this case, the underlying gauge theory is fundamental even in the presence of elementary scalars [54].

Having nonsupersymmetric, interacting, four-dimensional conformal field theories (CFTs) at our disposal, we determine the physical properties of the singlet $\operatorname{Tr}\left[\mathrm{HH}^{\dagger}\right]$ and the adjoint $\operatorname{Tr}\left[T^{a} H T^{a} H^{\dagger}\right]$ composite operators. Via an explicit computation, we discover that the singlet anomalous dimension is substantially larger than the adjoint one. We then construct the four-point correlations functions in which these operators play an important role, and check the crossing relations. Furthermore in the Veneziano limit, and at the maximum known order in perturbation theory, we argue that the singlet sector of the theory is nontrivial. We finally compare, when possible, our precise results with the numerical bootstrap constraints [105-107, 109, 110].

The work is organized as follows. In section 2 we briefly review the conformal bootstrap idea and the associated bounds [105-107]. We then move on to derive the conformal bootstrap sum rules in a CFT with non-Abelian global symmetry $\mathrm{SU}\left(N_{f}\right) \times \mathrm{SU}\left(N_{f}\right)$ in section 3 . The four dimensional gauge-Yukawa theories used here are introduced in section 4 . In the same section we also argue that the singlet sector decouples from the other operators. In section 5 , we offer our conclusions.

\section{Conformal bootstrap review}

To set the stage, we provide a short, self-contained introduction to the idea of the conformal bootstrap and highlight its salient properties. We consider the set of correlation functions for all local operators of some quantum field theory. For this to constitute a conformal field theory, the set of correlation functions must obey a corresponding set of constrains, and presently, we set out to find it. A CFT consists of its conformal primary operators $\mathcal{O}_{i}{ }^{1}$ and their associated conformal dimensions $\Delta_{i}$ and spins $l_{i}$. Because of conformality, the

\footnotetext{
${ }^{1}$ Primary operators are annihilated by generators of special conformal transformation $\left[K_{\mu}, \mathcal{O}(0)\right]=0$ where we inserted primary operator at $x=0$ point and $K_{\mu}$ denotes the generator of the special conformal transformation.
} 
normalization is completely arbitrary, and we select a basis for the scalar operators such that the 2-point functions have the form

$$
\left\langle\mathcal{O}_{i}(x) \mathcal{O}_{j}(y)\right\rangle=\frac{\delta_{i j}}{|x-y|^{2 \Delta_{i}}}
$$

$\Delta_{i}$ must satisfy the unitarity constraints [108]:

$$
\begin{array}{ll}
\Delta_{i} \geq 1 & \left(l_{i}=0\right) \\
\Delta_{i} \geq l_{i}+2 & \left(l_{i} \geq 1\right) .
\end{array}
$$

In any CFT, it is possible to express the product of two local operators as a sum over all local operators in the theory which have a finite radius of convergence. This is called the operator product expansion (OPE), and we have

$$
\mathcal{O}_{i}(x) \mathcal{O}_{j}(y)=\sum_{k} c_{i j}^{k}(x-y) \mathcal{M}_{k}(y)
$$

where, as mentioned, the sum is over all (primary and non-primary) local operators $\mathcal{M}_{k}$ and $c_{i j}^{k}(x-y)$ are functions of the dimensions and spins (which we denote collectively by the index $\left.k=\left(\Delta_{k}, l_{k}\right)\right)$ of the operators involved, and of the dynamics of the theory. Using equation (2.4) inside correlation functions, we can replace a product (like the l.h.s.) by a sum (like the r.h.s.), as long as there are no other operators at smaller distances from $y$ than $|x-y|$.

The OPE above is quite general, and by also imposing conformal invariance it can be shown [111] that the kinematics of the primary operators uniquely determines the coefficients $c_{i j}^{k}(x-y)$ belonging to their descendant operators. ${ }^{2}$ Thus, all dynamical information in the OPE is encoded in the coefficients for the primary operators

$$
\mathcal{O}_{i}(x) \mathcal{O}_{j}(y)=\sum_{k} C_{i j}^{k} \mathcal{O}_{k} \frac{1}{|x-y|^{\Delta_{i}+\Delta_{j}-\Delta_{k}}}+\text { descendants contribution, }
$$

where the new coefficients $C_{i j}^{k}$ are translation invariant constants. The complete OPE (with both primary and descendant contributions) is then

$$
\mathcal{O}_{i}(x) \mathcal{O}_{j}(y)=\sum_{k} C_{i j}^{k} L_{k}\left(x-y, \partial_{y}\right) \mathcal{O}_{k}(y) \frac{1}{|x-y|^{\Delta_{i}+\Delta_{j}-\Delta_{k}}}
$$

where $L_{k}\left(x-y, \partial_{y}\right)$ are differential operators that only depend on the kinematics, that is the dimensions and spins of the primary operators $\mathcal{O}_{k}$. They do not depend on the dynamics of the CFT. By using the OPE on the two operators that are closest together, it is now a straightforward matter to reduce an $n$-point function to an infinite sum over $(n-1)$-point functions, which in turn can be reduced to an infinite sum of $(n-2)$-point functions, and so on down to the 2-point functions, which have the simple structure seen in (2.1). Thus,

\footnotetext{
${ }^{2}$ The descendant operators are obtained by acting on the primaries with the translation operator i.e. taking derivatives of the primaries.
} 
if we know the conformal dimensions $\Delta_{i}$, the spins $l_{i}$ and the 3 -point coefficients $C_{i j}^{k}$ of the primary operators, we know the entire CFT.

If we have multiple operators, there are several ways of using the OPE to reduce an $n$-point function. However, this obviously cannot change the result, and thus we must insist that regardless of the order in which multiple OPE contractions are used, the end results must be equal. This leads to non-trivial constraints on the possible values of $\Delta_{i}$ and $C_{i j}^{k}$ that can make up a consistent CFT. For comprehensive review on both of these constraints see [107].

As an instructive example, we consider the 4-point function $\left\langle\mathcal{O}_{1}\left(x_{1}\right) \mathcal{O}_{2}\left(x_{2}\right) \mathcal{O}_{3}\left(x_{3}\right)\right.$ $\left.\mathcal{O}_{4}\left(x_{4}\right)\right\rangle$. We can evaluate this using the OPE between the operators at $x_{1}$ and $x_{2}$ and simultaneously at $x_{3}$ and $x_{4}$, or alternatively by performing the OPE between the operators at $x_{1}$ and $x_{4}$ and simultaneously at $x_{2}$ and $x_{3}$. This corresponds to the s-channel (12) $\rightarrow(34)$ and t-channel $(14) \rightarrow(23)$ respectively. ${ }^{3}$ The contraction in the s-channel yields

$$
\left\langle\mathcal{O}_{1}\left(x_{1}\right) \mathcal{O}_{2}\left(x_{2}\right) \mathcal{O}_{3}\left(x_{3}\right) \mathcal{O}_{4}\left(x_{4}\right)\right\rangle=\sum_{k} \frac{C_{12}^{k} C_{34}^{k} L_{k}\left(x_{12}, \partial_{x_{2}}\right) L_{k}\left(x_{34}, \partial_{x_{4}}\right)\left\langle\mathcal{O}_{k}\left(x_{2}\right) \mathcal{O}_{k}\left(x_{4}\right)\right\rangle}{\left|x_{12}\right|^{\Delta_{1}+\Delta_{2}-\Delta_{k}}\left|x_{34}\right|^{\Delta_{3}+\Delta_{4}-\Delta_{k}}}
$$

In this expression, only the OPE coefficients $C_{12}^{k}$ and $C_{34}^{k}$ depend on the dynamics of the CFT. It is therefore convenient to define the conformal blocks

$$
\begin{aligned}
\mathbf{G}_{k}^{12,34}\left(x_{1}, x_{2}, x_{3}, x_{4}\right) \equiv & \frac{1}{\left|x_{12}\right|^{\Delta_{1}+\Delta_{2}-\Delta_{k}}} \frac{1}{\left|x_{34}\right|^{\Delta_{3}+\Delta_{4}-\Delta_{k}}} \\
& \times L_{k}\left(x_{12}, \partial_{x_{2}}\right) L_{k}\left(x_{34}, \partial_{x_{4}}\right)\left\langle\mathcal{O}_{k}\left(x_{2}\right) \mathcal{O}_{k}\left(x_{4}\right)\right\rangle,
\end{aligned}
$$

which contain every contribution from the local operator $\mathcal{O}_{k}$ and its many descendants. As mentioned above, these conformal blocks are dependent only on the kinematics of the conformal group, and explicit expressions for them are given in [112, 113].

The above evaluation was done in the s-channel $(12) \rightarrow(34)$, but we could equally well have performed it in the t-channel $(14) \rightarrow(23)$. This would have given us a similar, but distinct, expression with 2 and 4 interchanged. Imposing that these two procedures give equal expressions is what yields the non-trivial conformal bootstrap equation

$$
\sum_{k} C_{12}^{k} C_{34}^{k} \mathbf{G}_{k}^{12,34}\left(x_{1}, x_{2}, x_{3}, x_{4}\right)=\sum_{k} C_{14}^{k} C_{23}^{k} \mathbf{G}_{k}^{14,23}\left(x_{1}, x_{4}, x_{2}, x_{3}\right),
$$

which, together with (2.8), tells us how the dimensions, spins and OPE coefficients must relate to each other in order for the theory in question to be conformal.

In addition, conformal symmetry allows us to further constrain the coordinate dependence of the 4-point function and the most general conformally invariant expression is

$$
\begin{aligned}
\left\langle\mathcal{O}_{1}\left(x_{1}\right) \mathcal{O}_{2}\left(x_{2}\right) \mathcal{O}_{3}\left(x_{3}\right) \mathcal{O}_{4}\left(x_{4}\right)\right\rangle & =\sum_{k} C_{12}^{k} C_{34}^{k} \mathbf{G}_{k}^{12,34}\left(x_{1}, x_{2}, x_{3}, x_{4}\right) \\
& \equiv\left(\frac{\left|x_{24}\right|}{\left|x_{14}\right|}\right)^{\Delta_{1}-\Delta_{2}}\left(\frac{\left|x_{14}\right|}{\left|x_{13}\right|}\right)^{\Delta_{3}-\Delta_{4}} \frac{g(u, v)}{\left|x_{12}\right|^{\Delta_{1}+\Delta_{2}}\left|x_{34}\right|^{\Delta_{3}+\Delta_{4}}},
\end{aligned}
$$

\footnotetext{
${ }^{3}$ It is also possible to make the contractions in the u-channel $(13) \rightarrow(24)$, but this gives no additional constraints.
} 
where $g(u, v)$ is an arbitrary function ${ }^{4}$ of the conformally-invariant cross-ratios:

$$
u=\frac{x_{12}^{2} x_{34}^{2}}{x_{13}^{2} x_{24}^{2}}, \quad v=\frac{x_{14}^{2} x_{23}^{2}}{x_{13}^{2} x_{24}^{2}} .
$$

In [105], the bootstrap equation for the 4-point function of four identical scalar operators $\langle\phi \phi \phi \phi\rangle$ was considered. Starting from the OPE:

$$
\phi(x) \phi(0)=\frac{1}{x^{2 d}}\left(1+C_{\phi \phi}|x|^{\Delta} \phi^{2}(0)+\ldots\right), \quad d \equiv \Delta_{\phi},
$$

and using (2.10) with all $\Delta_{i}=d$ equal, we obtain:

$$
\begin{aligned}
&\left\langle\phi\left(x_{1}\right) \phi\left(x_{2}\right) \phi\left(x_{3}\right) \phi\left(x_{4}\right)\right\rangle=\frac{g(u, v)}{x_{12}^{2 d} x_{34}^{2 d}}, \\
& g(u, v)=1+\sum p_{k} g_{k}(u, v), \quad p_{k} \equiv\left(C_{\phi \phi}^{k}\right)^{2} \geq 0,
\end{aligned}
$$

where we explicitly separated the contribution of the identity operator. The explicit expression for the conformal blocks $g_{k}(u, v)$ reads:

$$
\begin{aligned}
g_{k}(u, v) & =g_{\Delta, l}(u, v)=\frac{(-1)^{l}}{2^{l}} \frac{z \bar{z}}{z-\bar{z}}\left[k_{\Delta+l}(z) k_{\Delta-l-2}(\bar{z})-(z \leftrightarrow \bar{z})\right], \\
k_{\beta}(x) & \equiv x^{\beta / 2}{ }_{2} F_{1}(\beta / 2, \beta / 2, \beta ; x), \quad u=z \bar{z}, \quad v=(1-z)(1-\bar{z}),
\end{aligned}
$$

where ${ }_{2} F_{1}$ is Gauss's hypergeometric function.

The 4-point function on the left-hand side of eq. (2.13) is obviously symmetric under the interchange of any two $x_{i}$, and its conformal block decomposition (2.14) must therefore also respect this symmetry. Invariance with respect to $x_{1} \leftrightarrow x_{2}$ or $x_{3} \leftrightarrow x_{4}$ implies that only operators of even spin are exchanged. The non-trivial constraint comes from the symmetry with respect to $x_{1} \leftrightarrow x_{3}$ and gives the following condition (see figure 1 for an illustration)

$$
v^{d} g(u, v)=u^{d} g(v, u),
$$

which is not automatically satisfied for $g(u, v)$ as given in equation $(2.14) .{ }^{5}$

Following [105], it is useful to rewrite $(2.16)$ by separating the unit operator contribution, which gives

$$
u^{d}-v^{d}=\sum_{k} p_{k}\left[v^{d} g_{k}(u, v)-u^{d} g_{k}(v, u)\right]
$$

where the index $k$ covers the conformal dimension $\Delta$ and the spin $l$, as in (2.15). The l.h.s. of this equation is the imbalance created by the presence of the unit operator in the OPE. This imbalance has to be compensated by contributions of the other fields on the r.h.s..

\footnotetext{
${ }^{4}$ Note that we absorbed the OPE coefficients $C_{12}^{k}$ and $C_{34}^{k}$ into the definition of $g(u, v)$.

${ }^{5}$ The appearance of the $(u / v)^{d}$ factor in this relation is due to a nontrivial transformation of the prefactor $1 /\left(x_{12}^{2 d} x_{34}^{2 d}\right)$ in $(2.13)$.
} 


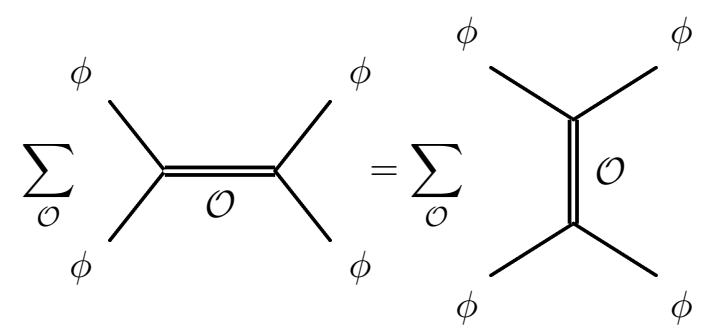

Figure 1. Diagrammatic expression of the conformal bootstrap equation. The double line denotes a conformal block, summing up exchanges of a primary operator $\mathcal{O}$ and all of its descendants.

In practice, it is convenient to normalize (2.17) by dividing both sides by $u^{d}-v^{d}$. The resulting sum rule takes the form:

$$
1=\sum_{k} p_{k} F_{d, k}, \quad F_{d, k} \equiv \frac{v^{d} g_{k}(u, v)-u^{d} g_{k}(v, u)}{u^{d}-v^{d}} .
$$

For a given spectrum of operator dimensions and spins $\{\Delta, l\}$ the sum rule (2.18) can be viewed as an equation for the coefficients $p_{\Delta, l} \geq 0$. If there are no solutions to this equation, the corresponding CFT would be ruled out.

To achieve a concrete realization of this idea, it is necessary to have a practical recipe to show that the solution does not exist. For a simple example of such recipe, imagine that a certain derivative, e.g. $\partial_{x}$, when applied to every $F_{d, \Delta, l}$ and evaluated at a certain point, is strictly positive. Since the same derivative applied to the l.h.s. of (2.18) gives identically zero, a solution where all coefficients $p_{\Delta, l}$ are non-negative would clearly be impossible. Using this logic, a first model-independent bound on the dimension of the operator $\phi^{2}$ was numerically found in $[105,106]$ by using linear programming methods:

$$
\Delta \leq \Delta_{\min }=2+0.7 \sqrt{d-1}+2.1(d-1)+0.43(d-1)^{3 / 2},
$$

where $d$ is the conformal dimension of the scalar $\phi, d \equiv \Delta_{\phi}$, and $\Delta$ is the dimension of the operator $\phi^{2}, \Delta \equiv \Delta_{\phi^{2}}$. In [107] a semidefinite programming algorithm was used and the bound was improved further to the current strongest limit:

$$
\Delta \leq \Delta_{\max }=2+3.006(d-1)+0.16\left(1-e^{-20(d-1)}\right) .
$$

There does not seem to be any known 4D unitary CFT saturating this bound.

\section{Conformal bootstrap sum rules in CFT with $\mathrm{SU}\left(N_{f}\right)_{L} \times \mathrm{SU}\left(N_{f}\right)_{R}$ global symmetry}

We will construct explicit examples of CFTs stemming from four-dimensional, nonsupersymmetric gauge-Yukawa theories possessing the global, non-Abelian symmetry $\mathrm{SU}\left(N_{f}\right)_{L} \times$ $\mathrm{SU}\left(N_{f}\right)_{R}$. For this reason, we will proceed to generalize the conformal block decomposition to this particular case since it has not, to our knowledge, previously been studied in the literature. Similar analyses have been carried out for the $\mathrm{SO}(N)$ and $\mathrm{SU}(N)$ cases in [114]. The 
relevant gauge singlet complex scalar degrees of freedom are bi-fundamental with respect to the $\mathrm{SU}\left(N_{f}\right)_{L} \times \mathrm{SU}\left(N_{f}\right)_{R}$ global symmetry and can be mathematically represented as:

$$
H_{i}^{\alpha^{*}}=\left(\mathbf{N}_{\mathbf{f}}, \mathbf{N}_{\mathbf{f}}^{*}\right) \quad \text { and } \quad H_{j^{*}}^{\beta}=\left(\mathbf{N}_{\mathbf{f}}^{*}, \mathbf{N}_{\mathbf{f}}\right),
$$

where all indices $i, j, \alpha, \beta=1,2, \ldots, N_{f}$. Latin indices are for $\mathrm{SU}\left(N_{f}\right)_{L}$ and Greek indices for $\mathrm{SU}\left(N_{f}\right)_{R}$ respectively. It is convenient to introduce the following matrix notation

$$
H_{i}^{\alpha^{*}}=(H)_{i \alpha} \quad \text { and } \quad H_{j^{*}}^{\beta}=\left(H^{\dagger}\right)_{\beta j} .
$$

We start with the OPE analysis for the following composite operator:

$$
\begin{aligned}
H_{i \alpha}(x) \times H_{\beta j}^{\dagger}(0) \sim & \frac{1}{|x|^{2 d_{H}}}\left\{\delta_{i j} \delta_{\alpha \beta}\left[1+c_{S}|x|^{\Delta_{S}} \operatorname{Tr}\left[H H^{\dagger}\right](0)\right]\right. \\
& \left.+c_{L}|x|^{\Delta_{L}} \delta_{i j} M_{k k \alpha \beta}(0)+c_{R}|x|^{\Delta_{R}} \delta_{\alpha \beta} M_{i j \gamma \gamma}(0)+c_{A}|x|^{\Delta_{A}} M_{i j \alpha \beta}(0)+\cdots\right\},
\end{aligned}
$$

where, in the free theory, $M_{i j \alpha \beta} \equiv H_{i \alpha} H_{\beta j}^{\dagger}-\frac{1}{N_{f}^{2}} \operatorname{Tr}\left[H H^{\dagger}\right] \delta_{i j} \delta_{\alpha \beta}$ and $d_{H}$ is the conformal dimension of the $H$ field. The group-theoretical content of the OPE above is:

$$
\left(\mathbf{N}_{\mathbf{f}}, \mathbf{N}_{\mathbf{f}}^{*}\right) \times\left(\mathbf{N}_{\mathbf{f}}^{*}, \mathbf{N}_{\mathbf{f}}\right)=(\mathbf{1}, \mathbf{1})+(\mathbf{1}, \operatorname{Adj})+(\operatorname{Adj}, \mathbf{1})+(\operatorname{Adj}, \operatorname{Adj}) .
$$

The crossing symmetry constraints are derived by equating the $(12) \rightarrow(34)$ and $(14) \rightarrow(23)$ s- and t-channel conformal block decompositions of the following 4-point function

$$
\left\langle H\left(x_{1}\right) H\left(x_{2}\right)^{\dagger} H\left(x_{3}\right) H\left(x_{4}\right)^{\dagger}\right\rangle=\left\langle H\left(x_{1}\right) H\left(x_{4}\right)^{\dagger} H\left(x_{3}\right) H\left(x_{2}\right)^{\dagger}\right\rangle .
$$

There are four basic invariants contained in $\left[H\left(x_{1}\right) \times H\left(x_{2}\right)^{\dagger}\right] \times\left[H\left(x_{3}\right) \times H\left(x_{4}\right)^{\dagger}\right]$. Using (3.4), we see that the overall singlet terms contributing are:

$$
\begin{aligned}
& \{[(\mathbf{1}, \mathbf{1})+(\mathbf{1}, \operatorname{Adj})+(\operatorname{Adj}, \mathbf{1})+(\operatorname{Adj}, \operatorname{Adj})] \times[(\mathbf{1}, \mathbf{1})+(\mathbf{1}, \operatorname{Adj})+(\operatorname{Adj}, \mathbf{1})+(\operatorname{Adj}, \text { Adj })]\}_{\text {singlet }} \\
& \quad=\mathbf{G}_{\mathbf{S}}(\mathbf{1}, \mathbf{1})+\mathbf{G}_{\mathbf{L}}\left(\mathbf{1}, \mathbf{1}_{\mathbf{A A}}\right)+\mathbf{G}_{\mathbf{R}}\left(\mathbf{1}_{\mathbf{A A}}, \mathbf{1}\right)+\mathbf{G}_{\mathbf{A}}\left(\mathbf{1}_{\mathbf{A A}}, \mathbf{1}_{\mathbf{A A}}\right)
\end{aligned}
$$

where $\mathbf{1}_{\mathbf{A A}}$ means that we have to extract the singlet from the tensor product of the two adjoint representations. In general, each of the four basic invariants $G_{S, L, R, A}$ contain operators of both even and odd spins.

We now derive the constraint stemming from crossing symmetry in terms of these four basic invariants. For the s- and t-channel conformal block decompositions we obtain:

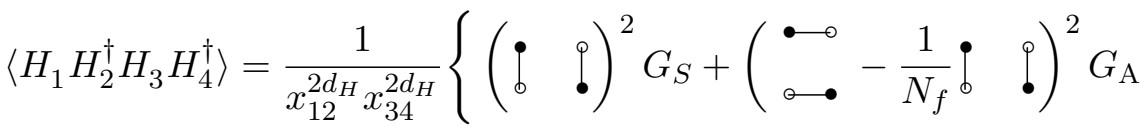

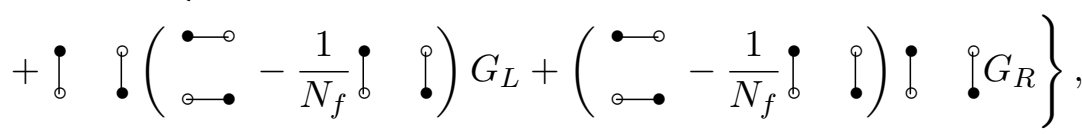

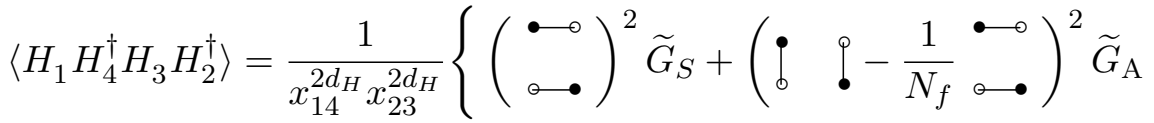

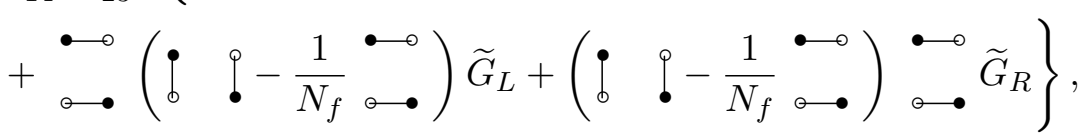


where $H_{i}=H\left(x_{i}\right), d_{H}$ is the quantum physical dimension of the $H$ field, $\widetilde{G} \equiv G_{u \leftrightarrow v}$ and we used a graphical notation for the tensor contractions. The squaring of the contractions $(\ldots)^{2}$ means that we have to perform the same contraction for both $\mathrm{SU}\left(N_{f}\right)$ factors. Every line means that the corresponding indices are contracted with the $\delta$-tensor:

$$
\left\lceil=\delta_{i j} \delta_{\alpha \beta}, \quad\right. \text { etc. }
$$

Now, equating the s- and t-channel decompositions and demanding that the coefficients multiplying the corresponding tensors match, we deduce:

$$
\begin{aligned}
& \left(\begin{array}{ll}
\bullet & \bullet
\end{array}\right)^{2}: v^{d_{H}}\left(G_{S}-\frac{1}{N_{f}}\left(G_{L}+G_{R}\right)+\frac{1}{N_{f}^{2}} G_{\mathrm{A}}\right)=u^{d_{H}} \widetilde{G}_{\mathrm{A}}, \\
& (\bullet)^{2}: v^{d_{H}} G_{\mathrm{A}}=u^{d_{H}}\left(\widetilde{G}_{S}-\frac{1}{N_{f}}\left(\widetilde{G}_{L}+\widetilde{G}_{R}\right)+\frac{1}{N_{f}^{2}} \widetilde{G}_{\mathrm{A}}\right) \text {, } \\
& \bullet \bullet ?: v^{d_{H}}\left(G_{R}-\frac{1}{N_{f}} G_{\mathrm{A}}\right)=u^{d_{H}}\left(\widetilde{G}_{\mathrm{L}}-\frac{1}{N_{f}} \widetilde{G}_{A}\right) \text {, } \\
& \bullet \quad \bullet: v^{d_{H}}\left(G_{L}-\frac{1}{N_{f}} G_{\mathrm{A}}\right)=u^{d_{H}}\left(\widetilde{G}_{\mathrm{R}}-\frac{1}{N_{f}} \widetilde{G}_{A}\right) \text {, }
\end{aligned}
$$

which yields four equations with four unknowns.

These equations generalize (2.16) to the theories possessing the non-abelian symmetry $\mathrm{SU}\left(N_{f}\right)_{L} \times \mathrm{SU}\left(N_{f}\right)_{R}$ and can be solved numerically. The 4-point function $\left\langle H\left(x_{1}\right) H\left(x_{2}\right)^{\dagger} H\left(x_{3}\right) H\left(x_{4}\right)^{\dagger}\right\rangle$ can also be expanded in the $(13) \rightarrow(24)$ u-channel. For completeness, in appendix A we derive the crossing symmetry constraints using this channel while in a follow-up study we will analyze the system (3.10)-(3.13).

\section{A four-dimensional calculable Gauge-Yukawa CFT}

We consider an $\mathrm{SU}\left(N_{c}\right)$ gauge theory with $N_{f}$ fundamental Dirac fermions $Q=\left(q, \widetilde{q}^{*}\right)$, $\ell$ adjoint Weyl fermions $\lambda$, and a gauge singlet complex scalar $H$ that transforms in the bifundamental representation of the $\mathrm{SU}\left(N_{f}\right)_{L} \times \mathrm{SU}\left(N_{f}\right)_{R}$ global symmetry of the theory. For the benefit of the reader, the field content and the quantum symmetries of the theory with $\ell=1$ are summarized in table 1 . The hermiticity property of $H$ is defined as $\left(H_{i}^{\alpha^{*}}\right)^{\dagger} \equiv$ $H_{\alpha^{*}}^{i}$ and the matrix $H$ may be decomposed in terms of $2 N_{f}^{2}$ real scalar fields as follows:

$$
H_{i}^{\alpha^{*}}=H_{i \alpha}=\frac{\phi+i \eta}{\sqrt{2 N_{f}}} \delta_{i \alpha}+\sum_{A=1}^{N_{f}^{2}-1}\left(h^{A}+i \pi^{A}\right) T_{i \alpha}^{A}
$$

where $T_{i \alpha}^{A}$ are the usual generalized Gell-Mann matrices. The fields $H$ and $H^{\dagger}$ can be contracted to form a singlet

$$
(\mathbf{1}, \mathbf{1})=\delta_{i j} \delta_{\alpha \beta} H_{i \alpha} H_{\beta j}^{\dagger}=\operatorname{Tr}\left[H H^{\dagger}\right],
$$




\begin{tabular}{|c|c|cccc|}
\hline Fields & {$\left[\mathrm{SU}\left(N_{c}\right)\right]$} & $\mathrm{SU}\left(N_{f}\right)_{L}$ & $\mathrm{SU}\left(N_{f}\right)_{R}$ & $\mathrm{U}(1)_{V}$ & $\mathrm{U}(1)_{A F}$ \\
\hline$\lambda$ & $\mathrm{Adj}$ & 1 & 1 & 0 & 1 \\
$q$ & $\square$ & $\square$ & 1 & $\frac{N_{f}-N_{c}}{N_{c}}$ & $-\frac{N_{c}}{N_{f}}$ \\
$\widetilde{q}$ & $\square$ & 1 & $\square$ & $-\frac{N_{f}-N_{c}}{N_{c}}$ & $-\frac{N_{c}}{N_{f}}$ \\
\hline$H$ & 1 & $\square$ & $\overline{1}$ & 0 & $\frac{2 N_{c}}{N_{f}}$ \\
$G_{\mu}$ & $\operatorname{Adj}$ & 1 & 1 & 0 & 0 \\
\hline
\end{tabular}

Table 1. Field content of the example. The first three fields are Weyl spinors in the $\left(\frac{1}{2}, 0\right)$ representation of the Lorentz group. $H$ is a complex scalar and $G_{\mu}$ is the gauge field. $\mathrm{U}(1)_{A F}$ is the extra Anomaly Free symmetry arising due to the presence of $\lambda$.

or an adjoint with respect to the right or left handed groups:

$$
(\mathbf{A d j}, \mathbf{1})=H_{i \alpha} H_{\alpha j}^{\dagger}=\left(H H^{\dagger}\right)_{i j} \quad \text { or } \quad(\mathbf{1}, \mathbf{A d j})=H_{i \alpha} H_{\beta i}^{\dagger}=\left(H H^{\dagger}\right)_{\alpha \beta} \text {, }
$$

while (Adj, Adj) can be formed as a tensor product.

The Lagrangian of the theory is

$$
\begin{aligned}
\mathcal{L}= & \operatorname{Tr}\left[-\frac{1}{2} F^{\mu \nu} F_{\mu \nu}+i \bar{\lambda} \not D \lambda+\bar{Q} i \not D Q+\partial_{\mu} H^{\dagger} \partial^{\mu} H+y_{H} \bar{Q} H Q\right] \\
& -u_{1}\left(\operatorname{Tr}\left[H H^{\dagger}\right]\right)^{2}-u_{2} \operatorname{Tr}\left[H H^{\dagger} H H^{\dagger}\right] .
\end{aligned}
$$

Here Tr refers to the trace over both color and flavor indices and $D_{\mu}$ is the usual covariant derivative. At the renormalizable level we have the double trace $\left(\operatorname{Tr}\left[H H^{\dagger}\right]\right)^{2}$ and the single trace $\operatorname{Tr}\left[H H^{\dagger} H H^{\dagger}\right]$ operators.

Throughout this section we will work with the rescaled couplings which enable a finite Veneziano limit of the theory at fixed $\ell$. That is, we let both $N_{c}, N_{f} \rightarrow \infty$ while keeping $x \equiv N_{f} / N_{c}$ fixed. The appropriately rescaled couplings are

$$
a_{g}=\frac{g^{2} N_{c}}{(4 \pi)^{2}}, \quad a_{H}=\frac{y_{H}^{2} N_{c}}{(4 \pi)^{2}}, z_{1}=\frac{u_{1} N_{f}^{2}}{(4 \pi)^{2}}, z_{2}=\frac{u_{2} N_{f}}{(4 \pi)^{2}} .
$$

This model was introduced in $[75,101]$ to investigate near-conformal dynamics and its impact on the spectrum of the theory. Special attention was paid to the appearance of a dilaton, the Goldstone boson associated with the breaking of conformal symmetry, and its properties. The model was further investigated at higher orders in [102], and the properties related to the a-theorem were considered in [103].

\subsection{Beta functions and Weyl consistency conditions}

In order to perform a four-dimensional comparison with the bootstrap bound, we start by providing a calculable CFT at the highest known perturbative order. Following previous 
studies $[103,104]$ the beta functions of the theory are

$$
\begin{aligned}
& \beta_{a_{g}}=-\frac{2}{3} a_{g}^{2}\left[11-2 \ell-2 x+(34-16 \ell-13 x) a_{g}+3 x^{2} a_{H}+\frac{81 x^{2}}{4} a_{g} a_{H}\right. \\
&\left.-\frac{3 x^{2}(7+6 x)}{4} a_{H}^{2}+\frac{2857+112 x^{2}-x(1709-257 \ell)-1976 \ell+145 \ell^{2}}{18} a_{g}^{2}\right], \\
& \beta_{a_{H}=} a_{H}\left[2(x+1) a_{H}-6 a_{g}+(8 x+5) a_{g} a_{H}+\frac{20(x+\ell)-203}{6} a_{g}^{2}\right. \\
& \\
&\left.-8 x z_{2} a_{H}-\frac{x(x+12)}{2} a_{H}^{2}+4 z_{2}^{2}\right], \\
& \beta_{z_{1}=} 4\left(z_{1}^{2}+3 z_{2}^{2}+4 z_{1} z_{2}+z_{1} a_{H}\right), \\
& \beta_{z_{2}=}\left(2\left(2 z_{2} a_{H}+4 z_{2}^{2}-x a_{H}^{2}\right) .\right.
\end{aligned}
$$

Here we have already assumed the Veneziano limit and $\ell$ is the number of $\mathrm{SU}\left(N_{c}\right)$ adjoint Weyl fermions of the theory. ${ }^{6}$ We used the results of [115-118] to determine the beta functions and anomalous dimensions of the gauge-Yukawa theories investigated here.

The perturbative gauge beta function is considered up to and including the three loop order, the Yukawa to two and the scalar quartic couplings to the first order. This is the proper way of organizing perturbation theory for a multiple coupling theory as shown in $[103,104]$. In fact this counting can be mathematically related to the Weyl consistency conditions unveiled in the pioneering work by Osborn [119] and demonstrated to be relevant also for the standard model in [103, 104]. These conditions require the different beta functions to be related across different loop orders. Mathematically these conditions read:

$$
\frac{\partial\left(\chi^{j k} \beta_{k}\right)}{\partial g_{i}}=\frac{\partial\left(\chi^{i m} \beta_{m}\right)}{\partial g_{j}}
$$

with

$$
\chi^{i j} \equiv \operatorname{diag}\left(\frac{N_{c}^{2}}{128 \pi^{2} a_{g}^{2}}, \frac{N_{f}^{2}}{384 \pi^{2} a_{H}}, 0, \frac{N_{f}^{2}}{192 \pi^{2}}\right),
$$

where $g_{i} \equiv\left(a_{g}, \alpha_{H}, z_{1}, z_{2}\right)$ refers to the couplings collectively. To help the reader identify the related terms, according to the Weyl conditions, across the different couplings, we color-coded them directly in the beta functions. It is clear that these conditions relate the two-loop coefficients in the gauge beta function with one-loop coefficients in the Yukawa beta function (red color) and the two-loop coefficients in the Yukawa beta function with the one-loop coefficients in the quartic beta function (blue and brown colors). Our perturbative interacting CFTs live at the fixed point (FP) identified by the simultaneous zeros of the previous beta functions, i.e. we need to solve for $\beta_{a_{g}}=\beta_{a_{H}}=\beta_{z_{1}}=\beta_{z_{2}}=0$. The study of the beta functions above allowing us to establish the existence of perturbative CFTs has been performed in $[54,103]$. We will investigate the explicit physical results stemming from the analysis of these beta functions in 4.4 while in appendix B.5 we review, for completeness, the leading finite $N_{f}$ corrections to the beta functions [102].

\footnotetext{
${ }^{6}$ In table 1 we assumed $\ell=1$.
} 


\subsection{Higgs anomalous dimensions}

The existence of a perturbative CFT permits us to determine the conformal dimensions of the $(1,1)$ singlet $\Delta_{S} \equiv 2+\gamma_{S}$ and of the (Adj,Adj) adjoint $\Delta_{A} \equiv 2+\gamma_{A}$ composite operators. For the reader's convenience, we recall how these dimensions enters the OPE (3.3):

$$
H_{i \alpha}(x) \times H_{\beta j}^{\dagger}(0) \sim \frac{1}{|x|^{2 d_{H}}}\left\{\delta_{i j} \delta_{\alpha \beta}\left[1+c_{S}|x|^{\Delta_{S}} \operatorname{Tr}\left[H H^{\dagger}\right](0)\right]+c_{A}|x|^{\Delta_{A}} M_{i j \alpha \beta}(0)+\cdots\right\} .
$$

To compute these anomalous dimensions, we add to the Lagrangian (4.4) two mass terms $m_{S}^{2} \operatorname{Tr}\left[H H^{\dagger}\right]$ and $m_{A}^{2} \operatorname{Tr}\left[T^{a} H T^{a} H^{\dagger}\right],{ }^{7}$ and use [120] to specialize the formulae given in [115] to the present case.

We know from the Weyl consistency conditions [103, 104] that the order to which beta functions are computed in a gauge-Yukawa theory is distinctly non-trivial, and we must therefore also take care to compute the anomalous dimensions of the composite operators, as well as the Higgs field, to the proper order. To find this, we consider that if two of the four external legs on a Feynman digram that contributes to the quartic beta function are joined together, the resulting diagram is a constituent of the anomalous dimension of the composite operators to one higher order in the loop expansion. We therefore conclude that the anomalous dimensions should be computed to two loop order.

Thus, for the Higgs field $H$, we have that the anomalous dimension is

$\gamma_{H} \equiv d_{H}-1=a_{H}+2 z_{2}^{2}\left(1+\frac{1}{N_{f}^{2}}\right)-\frac{3 x a_{H}^{2}}{2}+\frac{5 a_{g} a_{H}}{2}\left(1-\frac{x^{2}}{N_{f}^{2}}\right)+2 z_{1}^{2}\left(\frac{1}{N_{f}^{2}}+\frac{1}{N_{f}^{4}}\right)+\frac{8 z_{1} z_{2}}{N_{f}^{2}}$,

and for the singlet and the adjoint composite operators:

$$
\begin{aligned}
& \gamma_{A}=\gamma_{\operatorname{Tr}\left[T^{a} H T^{a} H^{\dagger}\right]} \equiv \Delta_{A}-2=2 \gamma_{H}+\frac{4 z_{1}}{N_{f}^{2}}-\frac{8 a_{H} z_{1}}{N_{f}^{2}}-4 z_{1}^{2}\left(\frac{2}{N_{f}^{2}}+\frac{6}{N_{f}^{4}}\right)-\frac{32 z_{1} z_{2}}{N_{f}^{2}}-24 \frac{z_{2}^{2}}{N_{f}^{2}} \\
& \gamma_{S}=\gamma_{\operatorname{Tr}\left[H H^{\dagger}\right]} \equiv \Delta_{S}-2=\gamma_{A}+4\left(z_{1}+2 z_{2}\right)-8 a_{H}\left(z_{1}+2 z_{2}\right)-24 z_{2}^{2}-\frac{16 z_{1}^{2}}{N_{f}^{2}}-\frac{64 z_{1} z_{2}}{N_{f}^{2}}
\end{aligned}
$$

In appendix B.5 we show, for completeness, the leading finite $N_{f}$ corrections to these anomalous dimensions.

Having precisely computed, for the first time, the anomalous dimensions of relevant composite operators in this theory, it would be interesting to compare them with the bootstrap analysis. Such a comparison is, however, hampered by the fact that the analytic bootstrap conditions, we derived for $\mathrm{SU}\left(N_{f}\right)_{L} \times \mathrm{SU}\left(N_{f}\right)_{R}$, have not yet been solved numerically, like it is instead the case for $\mathrm{SU}(N)$ or $\mathrm{SO}(N)$ global symmetries [107]. In this initial exploration we will use partial simplifications occurring in the Veneziano limit of the theory to compare our precise results with some of the existing numerical bounds.

\footnotetext{
${ }^{7}$ Using the $\mathrm{SU}(N)$ generator identity $T_{i j}^{a} T_{k l}^{a}=\frac{1}{2} \delta_{i l} \delta_{j k}-\frac{1}{2 N} \delta_{i j} \delta_{k l}$, it is easy to see that $\operatorname{Tr}\left[T^{a} H T^{a} H^{\dagger}\right]=$ $\frac{1}{2} \operatorname{Tr}[H] \operatorname{Tr}\left[H^{\dagger}\right]-\frac{1}{2 N} \operatorname{Tr}\left[H H^{\dagger}\right]=\frac{1}{2} M_{i j i j}$ and thus how it is related to $M_{i j \alpha \beta}$.
} 


\subsection{Bootstrap in the Veneziano limit}

Interestingly, in the Veneziano limit, the conformal dimension of the (Adj, Adj) operator factorizes $\Delta_{A}=2 d_{H}\left(\gamma_{A}=2 \gamma_{H}\right)$, suggesting that, to two-loop order and in the Veneziano limit, we can identify $M_{i j \alpha \beta}(0)$ in (4.12) with the operator

$$
M_{i j \alpha \beta}(0) \sim: H_{j \beta} H_{\alpha i}^{\dagger}:(0)
$$

where we define the normal-ordered product : : of two operators as the non-singular part of the OPE in the limit where the two space-time points are brought together. Because the anomalous dimension of the adjoint is twice that of the $H$ field, this sector of the theory enjoys properties resembling those of a generalized free scalar $H(x)$ with conformal dimension $d_{H}=1+\gamma_{H}$. Therefore the correlation functions involving the composite adjoint operator are disconnected and can be written as products of 2-point functions. For example, using (4.15):

$$
\left\langle H_{i \alpha}\left(x_{1}\right) H_{\beta j}^{\dagger}\left(x_{2}\right) M_{i j \alpha \beta}(y)\right\rangle=\left\langle H_{i \alpha}\left(x_{1}\right) H_{\alpha i}^{\dagger}(y)\right\rangle\left\langle H_{j \beta}(y) H_{\beta j}^{\dagger}\left(x_{2}\right)\right\rangle,
$$

we can compute the 4-point function using the basic 2-point function:

$$
\left\langle H_{i \alpha}(x) H_{\alpha i}^{\dagger}(0)\right\rangle=\frac{1}{|x|^{2 d_{H}}} .
$$

Moreover, since the 3-point function, defining the OPE coefficient $c_{A}$ in (4.12), is fixed $\left(\Delta_{1}=\Delta_{2}=d_{H}\right.$ and $\left.\Delta_{y}=\Delta_{A}=2 d_{H}\right)$

$$
\begin{aligned}
\left\langle H_{i \alpha}\left(x_{1}\right) H_{\beta j}^{\dagger}\left(x_{2}\right) M_{i j \alpha \beta}(y)\right\rangle & =\frac{c_{A}}{\left|x_{12}\right|^{\Delta_{1}+\Delta_{2}-\Delta_{y}}\left|x_{1 y}\right|^{\Delta_{1}+\Delta_{y}-\Delta_{2}}\left|x_{2 y}\right|^{\Delta_{2}+\Delta_{y}-\Delta_{1}}} \\
& =\frac{c_{A}}{\left|x_{1 y}\right|^{2 d_{H}}\left|x_{2 y}\right|^{2 d_{H}}}
\end{aligned}
$$

by comparing with (4.16) and using (4.17) we see that $c_{A}=1$.

The factorization property of the (Adj, Adj) operators allows us to compute $G_{A}$ and $\widetilde{G}_{A}$ to this order in perturbation theory and in the Veneziano limit. Indeed, to compute $G_{A}$, for example, we start with the general expressions (2.10) (with all $\Delta_{i}=d_{H}$ ) and using notation of (3.7) write:

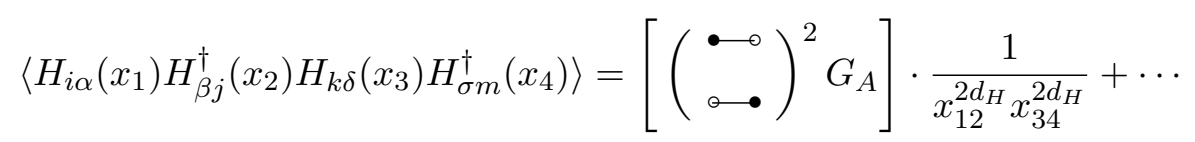

where we showed explicitly only the contributions from the conformal block $G_{A}$. As indicated by index contractions, we have to consider the correlator with external indices $(i=m, j=k)$ and $(\alpha=\sigma, \beta=\delta)$. Using the factorization property of (Adj,Adj), we 
calculate the $G_{A}$ contribution as follows $[121]^{8}$

$$
\left[\left\langle H_{i \alpha}\left(x_{1}\right) H_{\beta j}^{\dagger}\left(x_{2}\right) H_{k \delta}\left(x_{3}\right) H_{\sigma m}^{\dagger}\left(x_{4}\right)\right\rangle\right]_{G_{A}}=\frac{1}{x_{14}^{2 d_{H}} x_{23}^{2 d_{H}}}
$$

and therefore by comparing with (4.19) we deduce that:

$$
G_{A}=\left(\frac{u}{v}\right)^{d_{H}}=\frac{x_{12}^{2 d_{H}} x_{34}^{2 d_{H}}}{x_{14}^{2 d_{H}} x_{23}^{2 d_{H}}} .
$$

Similarly, for $\widetilde{G}_{A}$ we obtain $\widetilde{G}_{A}=(v / u)^{d_{H}}$. In terms of Feynman diagrams, factorization implies that the conformal block $G_{A}$ contributes only to the disconnected diagrams to this order in perturbation theory and in the Veneziano limit. These disconnected contributions provide the leading- $N_{f}$ dependence of the correlators which is known as large- $N_{f}$ factorization [122]. In fact, using the standard 't Hooft counting, it is easy to show that the disconnected contribution to our 4-point function in (4.21) appear at the $\mathcal{O}(1)$ while the fully connected contributions appear at the $\mathcal{O}\left(1 / N_{f}^{2}\right)$.

To take advantage of the large- $N_{f}$ factorization, following [121], we will be solving our bootstrap conditions (3.10)-(3.13) in the $1 / N_{f}$ expansion:

$$
\begin{aligned}
G_{S, A} & \equiv \sum_{\Delta, l} p_{\Delta, l}^{S, A} g_{\Delta, l}^{S, A}(u, v)=G_{S, A}^{\mathrm{disc}}+\frac{G_{S, A}^{\mathrm{conn}}}{N_{f}^{2}}+\cdots \\
G_{L, R} & \equiv \sum_{\Delta, l} p_{\Delta, l}^{L, R} g_{\Delta, l}^{L, R}(u, v)=\frac{G_{L, R}}{N_{f}}+\cdots
\end{aligned}
$$

where we formally divided the connected (conn) and disconnected (disc) contributions [121] to the conformal blocks $G_{S}$ and $G_{A}$. We also used the fact that $G_{L}$ and $G_{R}$ appear at the order $\mathcal{O}\left(1 / N_{f}\right)$ because they are disconnected with respect to just one of the two $\mathrm{SU}\left(N_{f}\right)$ factors.

At the leading $\mathcal{O}(1)$ in the large- $N_{f}$ expansion, from the bootstrap equations (3.10)(3.11) we have:

$$
\begin{array}{llll}
\mathcal{O}(1): & u^{d_{H}} \widetilde{G}_{A}^{\text {disc }}=v^{d_{H}} G_{S}^{\text {disc }}, & \text { with } & \widetilde{G}_{A}^{\text {disc }}=\left(\frac{v}{u}\right)^{d_{H}}, \\
\mathcal{O}(1): & v^{d_{H}} G_{A}^{\text {disc }}=u^{d_{H}} \widetilde{G}_{S}^{\text {disc }}, & \text { with } & G_{A}^{\text {disc }}=\left(\frac{u}{v}\right)^{d_{H}},
\end{array}
$$

which means that $G_{S}^{\text {disc }}=\widetilde{G}_{S}^{\text {disc }}=1$. These equations exemplify crossing symmetry constraints for the disconnected contributions to the 4-point function represented schematically in figure 2 .

\footnotetext{
${ }^{8}$ Here we assume a complete factorisation in the (Adj, Adj) channel although we have shown that it holds only for the leading operator. Therefore we have the generalized free Gaussian theory with OPE [121]:

$$
H\left(x_{1}\right) \times H^{\dagger}\left(x_{2}\right)=\frac{1}{x_{12}^{2 d_{H}}}+\sum_{n, l} \frac{c_{n, l}^{A}}{x_{12}^{n+l}} O_{n, l}^{A}
$$

where only double-trace operators $\mathcal{O}_{n, l}^{A}=\left(\mathcal{O}^{A} \overleftrightarrow{\partial_{\mu_{1}}} \ldots \overleftrightarrow{\partial_{\mu_{l}}}\left(\overleftrightarrow{\partial_{\nu}} \overleftrightarrow{\partial^{\nu}}\right)^{n} \mathcal{O}^{A}\right.$ - traces $)$ contribute. Here $l$ is the spin of the operator and $\Delta_{n, l}=2 d_{H}+2 n+l+\mathcal{O}\left(1 / N_{f}^{2}\right)$. The leading operator $O_{0,0}^{A}=\operatorname{Tr}\left[T^{a} H T^{a} H^{\dagger}\right]=$ $\frac{1}{2} \operatorname{Tr}[H] \operatorname{Tr}\left[H^{\dagger}\right]+\mathcal{O}\left(1 / N_{f}\right)$ has dimension $2 d_{H}$.
} 


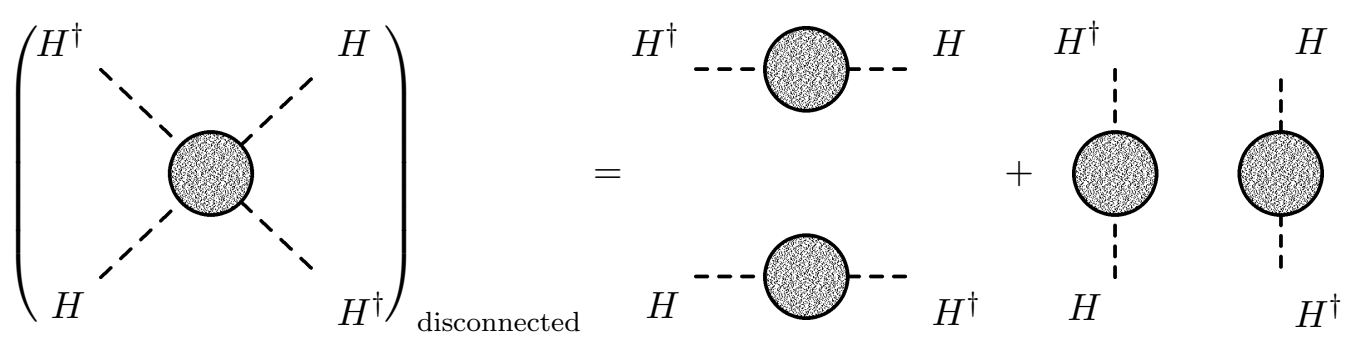

Figure 2. Disconnected contributions to the 4-point function.

The relevant bootstrap conditions at $\mathcal{O}\left(1 / N_{f}^{2}\right)$ are derived by combining the conditions (3.10)-(3.13) with the expansion (4.23)-(4.24), $G_{A}^{\text {disc }}=(u / v)^{d_{H}}, \widetilde{G}_{A}^{\text {disc }}=(v / u)^{d_{H}}$ and $G_{S}^{\text {disc }}=\widetilde{G}_{S}^{\text {disc }}=1$. By matching the $1 / N_{f}^{2}$ terms we have:

$$
\begin{array}{lc}
\mathcal{O}\left(1 / N_{f}^{2}\right): & v^{d_{H}}\left(G_{S}^{\mathrm{conn}}-\left(G_{L}+G_{R}\right)\right)+u^{d_{H}}=u^{d_{H}} \widetilde{G}_{A}^{\text {conn }}, \\
\mathcal{O}\left(1 / N_{f}^{2}\right): & u^{d_{H}}\left(\widetilde{G}_{S}^{\mathrm{conn}}-\left(\widetilde{G}_{L}+\widetilde{G}_{R}\right)\right)+v^{d_{H}}=v^{d_{H}} G_{A}^{\mathrm{conn}}, \\
\mathcal{O}\left(1 / N_{f}^{2}\right): & {\left[v^{d_{H}}\left(G_{L}+G_{R}\right)-u^{d_{H}}\left(\widetilde{G}_{L}+\widetilde{G}_{R}\right)\right]=2\left(u^{d_{H}}-v^{d_{H}}\right) .}
\end{array}
$$

The last equation is obtained by subtracting (3.13) from (3.12). Using (4.29) in the equation obtained by subtracting (4.28) from (4.27) we arrive at:

$$
v^{d_{H}} G_{S}^{\mathrm{conn}}-u^{d_{H}} \widetilde{G}_{S}^{\mathrm{conn}}=u^{d_{H}}\left(1+\widetilde{G}_{A}^{\mathrm{conn}}\right)-v^{d_{H}}\left(1+G_{A}^{\mathrm{conn}}\right) .
$$

Let us now consider the contributions to the conformal blocks $G_{S, A}^{\mathrm{conn}}$ and $\widetilde{G}_{S, A}^{\mathrm{conn}}$ more carefully. We will be using the work of [121] where the 4-point function of the singlet operators $\mathcal{O}(x)$ was considered. In this case, the lowest dimensional operator, aside from the unit operator, in the $\mathcal{O} \times \mathcal{O}$ OPE is the double trace operator $\mathcal{O}^{2}$ whose dimension $2 d[\mathcal{O}]+\mathcal{O}\left(1 / N_{f}^{2}\right)$ factorises at the lowest order in $1 / N_{f}$ expansion.

In our model, from (4.14), the dimensions of the adjoint operator satisfy the same factorization property and therefore the analysis of [121] applies. There it was shown that the conformal block $G_{A}^{\text {conn }}$ (and $\widetilde{G}_{A}^{\text {conn }}$ ) receives the contributions from the sum of two terms:

- $\mathcal{O}\left(1 / N_{f}^{2}\right)$ correction to the OPE coefficients $p_{\Delta, l}^{A}$. We will denote this contribution by $\left(G_{A}^{\text {conn }}\right)$ OPE

- $\mathcal{O}\left(1 / N_{f}^{2}\right)$ corrections to the anomalous dimension $\Delta_{A}$ which enter the functions $g_{\Delta, l}^{A}(u, v)$. We will denote this contribution by $\left(G_{A}^{\text {conn }}\right)^{A D}$.

The connected contribution to the conformal block can thus be expanded [121]:

$$
G_{A}^{\mathrm{conn}}=\left(G_{A}^{\mathrm{conn}}\right)^{\mathrm{OPE}}+\left(G_{A}^{\mathrm{conn}}\right)^{A D} .
$$

Furthermore, in our model the anomalous dimension for the singlet operator from (4.14) equals the anomalous dimension of the adjoint plus an additional non-factoriziable contribution $4\left(z_{1}+2 z_{2}\right)-8 a_{H}\left(z_{1}+2 z_{2}\right)-24 z_{2}^{2}$ and two additional $\mathcal{O}\left(1 / N_{f}^{2}\right)$ terms $-16 z_{1}^{2} / N_{f}^{2}-$ 
$64 z_{1} z_{2} / N_{f}^{2}$ not present in the anomalous dimensions for the adjoint (4.14). The nonfactorizable contribution is not present in the analysis of [121] and it will be taken into account.

Based on the above discussion it seems reasonable, but should still be proven, that $G_{A}^{\text {conn }}$ matches the factorazible part of $G_{S}^{\text {conn }}$. Assuming that this is true, the contributions due to the conformal blocks $\left(\left(G_{S, A}^{\text {conn }}\right)^{A D},\left(\widetilde{G}_{S, A}^{\text {conn }}\right)^{A D}\right)$ and $\left(\left(G_{S, A}^{\text {conn }}\right)^{\mathrm{OPE}},\left(\widetilde{G}_{S, A}^{\text {conn }}\right)^{\mathrm{OPE}}\right)$ cancel out in (4.30):

$$
\begin{gathered}
v^{d_{H}}\left(\left(G_{S}^{\text {conn }}\right)^{A D}+\left(G_{S}^{\text {conn }}\right)^{\mathrm{OPE}}\right)-u^{d_{H}}\left(\left(\widetilde{G}_{S}^{\text {conn }}\right)^{A D}+\left(\widetilde{G}_{S}^{\text {conn }}\right)^{\mathrm{OPE}}\right)= \\
u^{d_{H}}\left(\left(\widetilde{G}_{A}^{\text {conn }}\right)^{A D}+\left(\widetilde{G}_{A}^{\text {conn }}\right)^{\mathrm{OPE}}\right)-v^{d_{H}}\left(\left(G_{A}^{\text {conn }}\right)^{A D}+\left(G_{A}^{\text {conn }}\right)^{\mathrm{OPE}}\right) .
\end{gathered}
$$

The non-factorizable contribution to the singlet anomalous dimension quantifies the departure from the Gaussian limit and stems from an additional part of the singlet conformal block $\left(G_{S}^{\text {conn }}\right)^{\text {non-fact }}$. This part will not be balanced by an appropriate term associated with the adjoint composite operator in (4.30). This leads to a suggestive bootstrap equation for the non-factorizable part of the singlet

$$
v^{d_{H}}\left(G_{S}^{\text {conn }}\right)^{\text {non-fact }}-u^{d_{H}}\left(\widetilde{G}_{S}^{\text {conn }}\right)^{\text {non-fact }}=u^{d_{H}}-v^{d_{H}},
$$

which has precisely the form of (2.17), and we can even expand the conformal block in functions of the kinematics of the CFT $\left(G_{S}^{\text {conn }}\right)^{\text {non-fact }}=\sum p_{\Delta, l}^{S, n f} g_{\Delta, l}^{S, n f}(u, v)$.

Just as in (2.17), the right-hand side of (4.33) is the contribution from the Gaussian part of the theory which is balanced by the left-hand side. Therefore it would be tempting to interpret this result as a bound for $\Delta_{S}$, similar to the bound on the lowest dimensional operators of the theory coming from eq. (2.17), though holding only to the next-to-leading order in the couplings and in the Veneziano limit. This would mean that for low values of the couplings and high values of $N_{f}, \Delta_{S}$ should obey the bound given by eq. (2.20) with $d=d_{H}$.

However, there are several caveats to this suggestive statement that require further investigation. The most pressing is that our expression holds only for a part of $G_{S}$, and it is not clear how (or even if) a consistency equation on such a part would translate into a bound on $\Delta_{S}$. Another concern is that, as pointed out in [121], unitarity only implies positivity of $p_{\Delta, l}$ to leading order in $1 / N_{f}$, and if the expansion parameters $p_{\Delta, l}^{S, n f}$ are allowed to take either sign, the large $N_{f}$ analogue of the proof provided in [105] would be affected. Finally, the function $G_{S}$ contains an implicit sum over even and odd spins, while the bound of (2.20) is obtained for real scalars, where only even spins enter the crossing symmetry constraint.

The bound of [107] applies directly to the adjoint composite operator, the lowest dimensional operator, without any caveats and as we shall see in the specific examples provided below, the bound is well satisfied. As for the singlet composite operator, a direct comparison with the bound (2.20) quantifies the extent to which the caveats described above are under theoretical control. We stress again that a proper comparison requires a dedicated numerical bootstrap analysis for this theory.

In the examples below, we will also see that the anomalous dimension of the singlet composite operator can be substantially larger than that of the adjoint composite operator. 
Given that the bound on the singlet is unknown, this is a welcome feature which has been long sought after for nonperturbative models of near conformal dynamics used to describe composite Higgs scenarios, see [55] for a recent review.

\subsection{Physical results}

Now we review the salient points behind the existence of perturbative interacting CFTs [54, $103]$ and then determine the physical dimensions of the composite operators at the FPs of the theory.

The two-loop gauge beta function has a perturbative Banks-Zaks FP if the one-loop coefficient $b_{0}$ of the gauge beta function is small and the signs of the one-loop $b_{0}$ and two-loop $b_{1}$ coefficients are opposite. Therefore, our first task is to find a region in the parameter space of the model where the BZ FP exists. Solving (4.7) to one-loop and substituting into (4.6) we obtain

$$
b_{0}=\frac{2}{3}(11-2(\ell+x)), \quad b_{1}=\frac{2}{3}\left((34-16 \ell-13 x)+\frac{9 x^{2}}{x+1}\right) .
$$

From the asymptotic freedom $(\mathrm{AF})$ boundary condition $b_{0}=0$, we obtain $x=(11-2 \ell) / 2$. After substituting this value of $x$ into $b_{1}$

$$
b_{1 A F}=-\frac{25}{2}-\ell-\frac{3(11-2 \ell)^{2}}{4 \ell-26},
$$

we observe that for the unphysical value $\ell^{*} \approx 0.37$ the coefficient $b_{1 A F}$ vanishes. For $\ell=1$ we have that $b_{1 A F}$ is negative and for $\ell=0$ it is positive. Therefore in the first case we have an infrared BZ FP, and in the second we have an ultraviolet BZ FP. Note also [103] that in the absence of the Yukawa interactions the coefficient $b_{1 A F}$ in (4.35) is always negative and therefore the physical BZ FP can lead only to an infrared FP.

We are now ready to present our results for the $\ell=0$ and $\ell=1$ cases. Our strategy is the following

- For a given FP in all the couplings $\left(a_{g}^{*}, a_{H}^{*}, z_{1}^{*}, z_{2}^{*}\right)$ at a given value of $x \equiv N_{f} / N_{c}$, we determine the anomalous dimensions for the composite operators $\gamma_{S}$ and $\gamma_{A}$. We also determine the associated anomalous dimension of the scalar field $\gamma_{H}$. These results were obtained by means of the equations (4.13) and (4.14).

- We then insert $d=d_{H}=1+\gamma_{H}$ in the right hand-side of (2.19) and (2.20) to determine $\Delta_{\max }-2$. Finally, we compare the result with $\gamma_{A}$, which turns out to be the operator for which the bootstrap bound applies in all cases under consideration, and display $\gamma_{S}$, which is a more interesting quantity for phenomenology.

\subsubsection{The $\ell=0$ case}

The asymptotic freedom boundary, where the first coefficient of the gauge beta function vanishes, $b_{0}=0$, occurs at $x_{A F}=(11-2 \ell) / 2=5.5$. Increasing $x>x_{A F}$ slightly results in the appearance of an ultraviolet BZ FP, see figure 3a. An in depth analysis of the FP structure and its theoretical and phenomenological consequences for the asymptotic safety scenario has just appeared in [54]. 


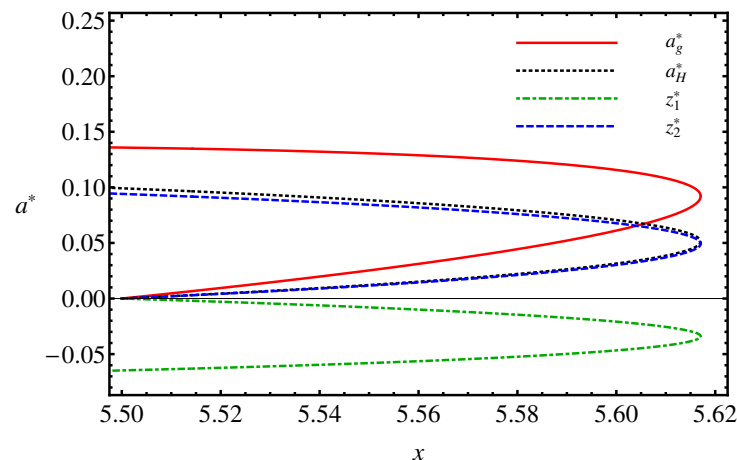

(a) Fixed point structure of the model with $\ell=0$. The boundary of asymptotic freedom is on the lefthand edge of the plot at $x=5.5$, the FP value of $a_{g}$ is the solid red line, $a_{H}$ is the dotted black, $z_{1}$ is the dot-dashed green, and $z_{2}$ is the dashed blue.

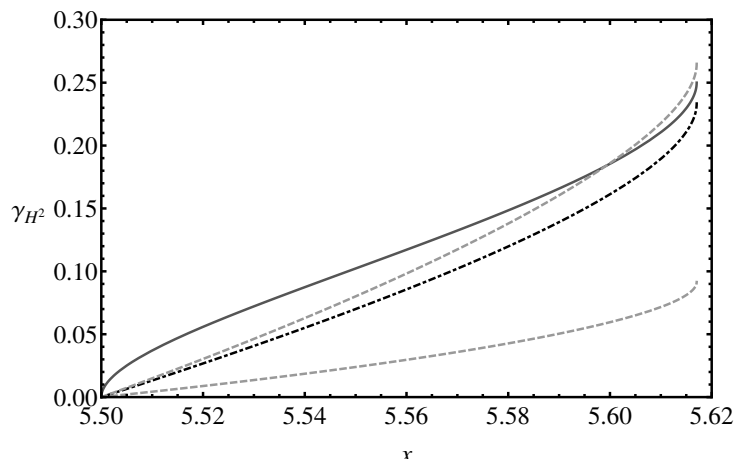

(b) Comparison with the conformal bootstrap bounds. The solid line is the bound (2.19), the dotdashed black is the revised bound (2.20), the dashed gray line is $\gamma_{\operatorname{Tr}\left[H^{\dagger}\right]}(4.14 \mathrm{~b})$, and the dotted light gray line is $\gamma_{\operatorname{Tr}\left[T^{a} H T^{a} H^{\dagger}\right]}(4.14 \mathrm{a})$.

Figure 3. FP structure and comparison with the bootstrap bound for the model with $\ell=0$.

When the three-loop gauge beta function is considered, an infrared FP emergers along with the ultraviolet $\mathrm{BZ} \mathrm{FP}$ in the range $x \leq x^{*} \approx 5.617$. At $x^{*}$ the ultraviolet $\mathrm{BZ} \mathrm{FP}$ and the infrared FP collide and both fixed points disappear. Perturbation theory is, of course, valid only for values of $\left(x-x_{A F}\right) / x_{A F} \ll 1$. As shown in [54] perturbation theory is valid for $\left(x-x_{A F}\right) / x_{A F}<0.1$.

The comparison with the bootstrap bound is shown in figure $3 \mathrm{~b}$. We first note that as expected, the bound is clearly respected by the anomalous dimension of the adjoint operator. More interestingly, we discover that the anomalous dimension of the singlet composite operator $\gamma_{S}$ is substantially larger than the anomalous dimension of the adjoint operator. If this also holds in the non-perturbative regime, this has important and welcome implications for model building.

\subsubsection{The $\ell=1$ case}

When the model is expanded to include adjoint fermions, the infrared BZ FP originates just below the asymptotic freedom boundary $x_{A F}=(11-2 \ell) / 2=4.5$ as shown in figure 4 a. The comparison of the composite operator anomalous dimensions with the two numerical bootstrap bounds is shown in figure $4 \mathrm{~b}$. As explained above, $\gamma_{A}$ is consistently below the bound in the perturbative regime. As for the $\ell=0$ case we determine the relevant quantity $\gamma_{S}$ and show that it is, also in this case, substantially larger than the adjoint operator.

\section{Conclusions}

We provided a systematic investigation of interesting properties of relevant composite operators stemming from gauge-Yukawa theories developing conformal fixed points in four dimensions. These theories are structurally similar to the standard model of particle interactions and have already been employed for interesting model building [40]. Having at 


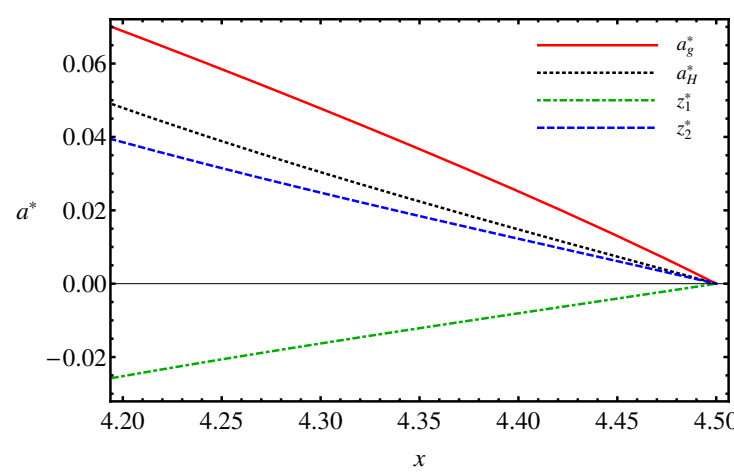

(a) Fixed point structure of the model with $\ell=1$. The FP value of $a_{g}$ is the solid red line, $a_{H}$ is the dotted black, $z_{1}$ is the dot-dashed green, and $z_{2}$ is the dashed blue.

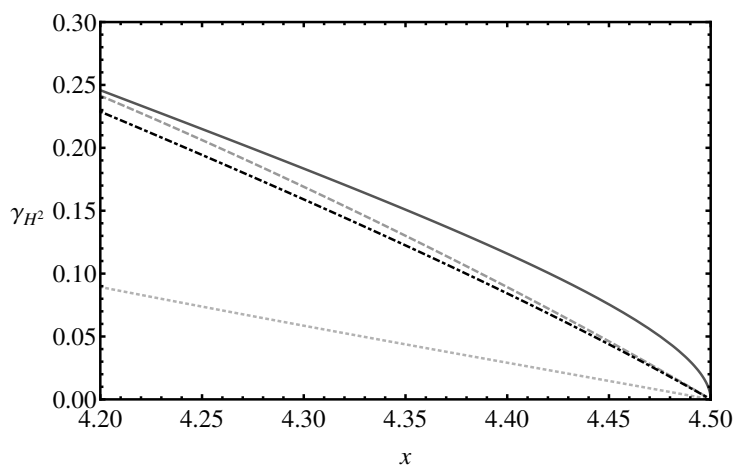

(b) Comparison of the conformal bootstrap bound. The solid gray line is the bound (2.19), the dotdashed black is the revised bound (2.20), the dashed gray line is $\gamma_{\operatorname{Tr}\left[H^{\dagger}\right]}(4.14 \mathrm{~b})$, and the dotted light gray line is $\gamma_{\operatorname{Tr}\left[T^{a} H T^{a} H^{\dagger}\right]}$ (4.14a).

Figure 4. FP structure and comparison with the bootstrap bound for the model with $\ell=1$.

our disposal explicit examples of nonsupersymmetric interacting four-dimensional CFTs, we investigated the critical exponents (anomalous dimensions at fixed points) associated to singlet $\operatorname{Tr}\left[H H^{\dagger}\right]$ and the adjoint $\operatorname{Tr}\left[T^{a} H T^{a} H^{\dagger}\right]$ operators.

We showed that in the Veneziano limit, and at the maximum known order in perturbation theory, the adjoint composite operator is Gaussian and automatically obeys the bootstrap bounds on the anomalous dimension. We also discovered that the singlet composite operator anomalous dimension at the interacting FP is substantially larger than the one for the adjoint composite operator. This is an interesting observation for phenomenologically driven questions regarding the possibility of large anomalous dimensions for singlet operators needed, for example, in theories of composite Higgs dynamics [55]. It would be interesting to analyze more generally the full bootstrap equations for these patterns of chiral symmetry.

Our results demonstrate the relevance of constructing conformal nonsupersymmetric four dimensional gauge-Yukawa theories that can be used for demonstrating the existence of four dimensional asymptotically safe theories [54], for interesting model building [40], probing the a-theorem [103], but also to either accurately test numerical solutions of the bootstrap constraints or determine novel anomalous dimensions of relevant composite operators. Following the pioneering work of Seiberg [123] it would be interesting to explore whether the weakly coupled four dimensional gauge-Yukawa theories investigated here have strongly coupled duals [124, 125].

\section{Acknowledgments}

We thank V. Rychkov for constructive discussions and insights. We also thank M. Redi for valuable discussions. The $\mathrm{CP}^{3}$-Origins centre is partially funded by the Danish National Research Foundation, grant number DNRF90. The work of OA is supported by the MIURFIRB grant RBFR12H1MW. 


\section{A The u-channel sum rule}

To extract the full information on the 4-point function, we also need to consider the $(13) \rightarrow(24) u$-channel OPE which reads:

$$
\mathbf{H} \times \mathbf{H}=\left(\mathbf{N}_{\mathbf{f}}, \mathbf{N}_{\mathbf{f}}^{*}\right) \times\left(\mathbf{N}_{\mathbf{f}}, \mathbf{N}_{\mathbf{f}}^{*}\right)=\left(\mathbf{2} \mathbf{S}, \mathbf{2} \mathbf{S}^{*}\right)^{+}+\left(\mathbf{2} \mathbf{A}, \mathbf{2} \mathbf{A}^{*}\right)^{+}+\left(\mathbf{2} \mathbf{S}, \mathbf{2} \mathbf{A}^{*}\right)^{-}+\left(\mathbf{2} \mathbf{A}, \mathbf{2} \mathbf{S}^{*}\right)^{-}
$$

where $2 S$ and $2 A$ stand for the two-index symmetric and antisymmetric tensors respectively. Due to the permutation symmetry of the $\mathbf{H H}$ state, the tensors $\left(\mathbf{2} \mathbf{S}, \mathbf{2} \mathbf{S}^{*}\right)^{+}$and $\left(\mathbf{2} \mathbf{A}, \mathbf{2} \mathbf{A}^{*}\right)^{+}$contain only even spins while the tensors $\left(\mathbf{2} \mathbf{S}, \mathbf{2} \mathbf{A}^{*}\right)^{-}$and $\left(\mathbf{2} \mathbf{A}, \mathbf{2} \mathbf{S}^{*}\right)^{-}$contain only odd spins.

As discussed in [114], the additional crossing symmetry constraints can be derived by equating the $(12) \rightarrow(34)$ and $(14) \rightarrow(23)$ s- and t-channel conformal block decompositions of the "transposed" $\left(H\left(x_{3}\right) \rightarrow H\left(x_{3}\right)^{\dagger}\right.$ and $\left.H\left(x_{4}\right)^{\dagger} \rightarrow H\left(x_{4}\right)\right)$ 4-point function $\left\langle H\left(x_{1}\right) H\left(x_{2}\right)^{\dagger} H\left(x_{3}\right)^{\dagger} H\left(x_{4}\right)\right\rangle$ :

$$
\left\langle H\left(x_{1}\right) H\left(x_{2}\right)^{\dagger} H\left(x_{3}\right)^{\dagger} H\left(x_{4}\right)\right\rangle=\left\langle H\left(x_{1}\right) H\left(x_{4}\right) H\left(x_{2}\right)^{\dagger} H\left(x_{3}\right)^{\dagger}\right\rangle .
$$

Now in the t-channel we have the same OPE as we would have in the u-channel of the original 4-point function and in the s-channel we have the same OPE up to the transposition of the fields at spacetime points $x_{3}$ and $x_{4}$. This transposition is taken into account by reversing the signs of the odd-spins contributions and permuting the flavor indices accordingly.

There are four basic invariants contained in the t-channel of the "transposed" 4-point function $\left[H\left(x_{1}\right) \times H\left(x_{4}\right)\right] \times\left[H\left(x_{2}\right)^{\dagger} \times H\left(x_{3}\right)^{\dagger}\right]$. Using (A.1), we see that the overall singlet terms contributing are:

$$
\begin{aligned}
& {\left[\left[\left(2 S, 2 S^{*}\right)+\left(2 A, 2 A^{*}\right)+\left(2 S, 2 A^{*}\right)+\left(2 A, 2 S^{*}\right)\right]\right.} \\
& \left.\quad \times\left[\left(2 S^{*}, 2 S\right)+\left(2 A^{*}, 2 A\right)+\left(2 S^{*}, 2 A\right)+\left(2 A^{*}, 2 S\right)\right]\right]_{\text {singlet }} \\
& =\mathbf{G}_{\mathbf{2} \mathbf{S}, \mathbf{2} \mathbf{S}}+\mathbf{G}_{\mathbf{2} \mathbf{A}, \mathbf{2} \mathbf{A}}+\mathbf{G}_{\mathbf{2} \mathbf{A}, \mathbf{2} \mathbf{S}}+\mathbf{G}_{\mathbf{2} \mathbf{S}, \mathbf{2} \mathbf{A}}
\end{aligned}
$$

where $\mathbf{1}_{\mathbf{2 S}}$ and $\mathbf{1}_{\mathbf{2 A}}$ means that we have to extract the singlet from the tensor product of the corresponding two-index representations.

From the $s=t$ channel crossing symmetry constrain of the "transposed" 4-point function we obtain:

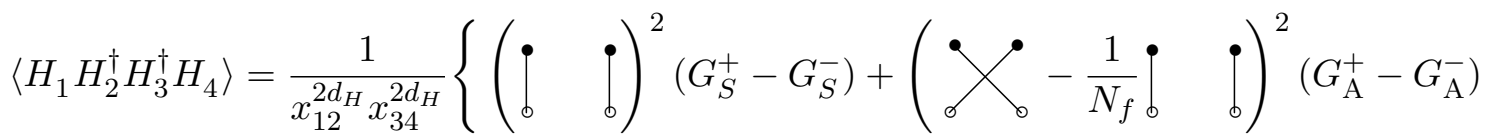

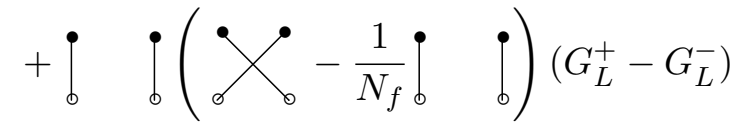

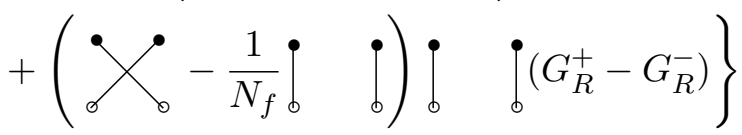




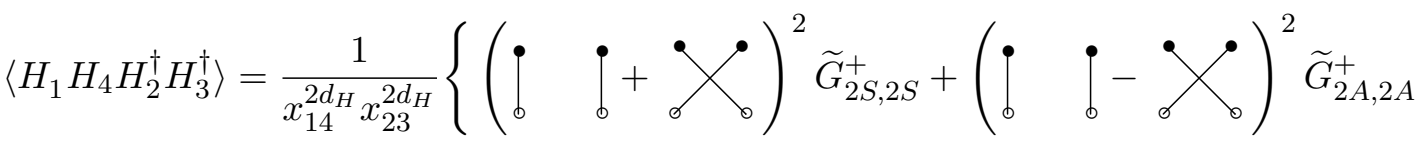

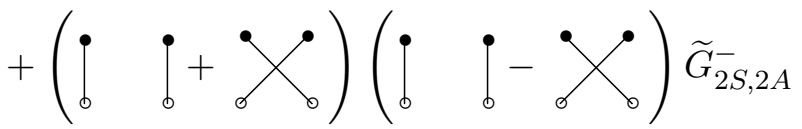

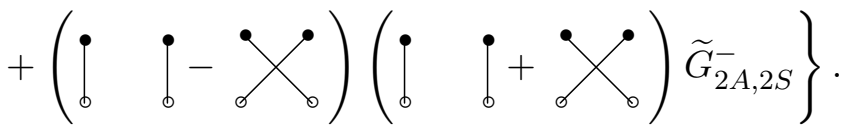

The s-channel decomposition is obtained from the previous case by transposing the index structure and flipping the sign of the odd-spin contributions. The t-channel decomposition is obtained by using the second OPE (A.1). The index structure is fixed by (anti)symmetry of the exchanged fields. Now, equating the s- and t-channel decompositions and demanding that the coefficients multiplying the corresponding tensors match we deduce:

$$
\begin{aligned}
& \left(\begin{array}{ll}
\bullet & \bullet
\end{array}\right)^{2}: v^{d_{H}}\left(G_{S}^{+}-G_{S}^{-}+\frac{1}{N_{f}^{2}}\left(G_{\mathrm{A}}^{+}-G_{\mathrm{A}}^{-}\right)-\frac{1}{N_{f}}\left(G_{L}^{+}-G_{L}^{-}+G_{R}^{+}-G_{R}^{-}\right)\right)= \\
& u^{d_{H}}\left(\widetilde{G}_{2 S, 2 S}^{+}+\widetilde{G}_{2 A, 2 A}^{+}+\widetilde{G}_{2 S, 2 A}^{-}+\widetilde{G}_{2 A, 2 S}^{-}\right) \\
& \left(\searrow_{0}\right)^{2}: v^{d_{H}}\left(G_{\mathrm{A}}^{+}-G_{\mathrm{A}}^{-}\right)=u^{d_{H}}\left(\widetilde{G}_{2 S, 2 S}^{+}+\widetilde{G}_{2 A, 2 A}^{+}-\widetilde{G}_{2 S, 2 A}^{-}-\widetilde{G}_{2 A, 2 S}^{-}\right) \\
& \sum_{0} i v^{d_{H}}\left(G_{R}^{+}-G_{R}^{-}-\frac{1}{N_{f}}\left(G_{\mathrm{A}}^{+}-G_{\mathrm{A}}^{-}\right)\right)=u^{d_{H}}\left(\widetilde{G}_{2 S, 2 S}^{+}-\widetilde{G}_{2 A, 2 A}^{+}+\widetilde{G}_{2 S, 2 A}^{-}-\widetilde{G}_{2 A, 2 S}^{-}\right) \\
& \text {† } \sum_{0}: v^{d_{H}}\left(G_{L}^{+}-G_{L}^{-}-\frac{1}{N_{f}}\left(G_{\mathrm{A}}^{+}-G_{\mathrm{A}}^{-}\right)\right)=u^{d_{H}}\left(\widetilde{G}_{2 S, 2 S}^{+}-\widetilde{G}_{2 A, 2 A}^{+}-\widetilde{G}_{2 S, 2 A}^{-}+\widetilde{G}_{2 A, 2 S}^{-}\right) \text {. }
\end{aligned}
$$

Working to lowest order in $1 / N_{f}$ and concentrating on the even spins conformal blocks $G_{2 S, 2 S}^{+}$and $G_{2 A, 2 A}^{+}$we have:

$$
v^{d_{H}}\left(G_{S}^{+}+G_{A}^{+}\right)=2 u^{d_{H}}\left(\widetilde{G}_{2 S, 2 S}^{+}+\widetilde{G}_{2 A, 2 A}^{+}\right) .
$$

\section{B Complete beta functions and anomalous dimensions beyond the Veneziano limit}

We provide here the full beta functions and anomalous dimensions of the gauge-Yukawa system. The conventions are the ones given in the main text.

For the beta functions we have:

$$
\begin{aligned}
\beta_{a_{g}}= & -\frac{2}{3} a_{g}^{2}\left[11-2 x-2 \ell+a_{g}\left(\frac{3 x^{3}}{N_{f}^{2}}-13 x-16 \ell+34\right)+3 x^{2} a_{H}\right. \\
& +a_{g} a_{H}\left(\frac{81 x^{2}}{4}-\frac{9 x^{4}}{4 N_{f}^{2}}\right)+a_{g}^{2}\left\{\frac{3 x^{5}}{4 N_{f}^{4}}-\frac{11 x^{3}(2 x+2 \ell-17)}{12 N_{f}^{2}}\right. \\
& \left.\left.+\frac{1}{18}\left(112 x^{2}+x(257 \ell-1709)+145 \ell^{2}-1976 \ell+2857\right)\right\}-\frac{3}{4}(6 x+7) x^{2} a_{H}^{2}\right]
\end{aligned}
$$




$$
\begin{aligned}
\beta_{a_{H}}= & 2 a_{H}\left[a_{g}\left(\frac{3 x^{2}}{N_{f}^{2}}-3\right)+(x+1) a_{H}+a_{g}^{2}\left(-\frac{3 x^{4}}{4 N_{f}^{4}}-\frac{x^{2}(5 x+5 \ell-53)}{3 N_{f}^{2}}+\frac{1}{12}(20 x+20 \ell-203)\right)\right. \\
& +a_{g} a_{H}\left(-\frac{(8 x+5) x^{2}}{2 N_{f}^{2}}+4 x+\frac{5}{2}\right)+a_{H}^{2}\left(\frac{2 x^{2}}{N_{f}^{2}}-\frac{1}{4} x(x+12)\right) \\
& \left.-\frac{8 x z_{1} a_{H}}{N_{f}^{2}}+z_{2} a_{H}\left(-\frac{4 x}{N_{f}^{2}}-4 x\right)+z_{1}^{2}\left(\frac{2}{N_{f}^{2}}+\frac{2}{N_{f}^{4}}\right)+\frac{8 z_{1} z_{2}}{N_{f}^{2}}+z_{2}^{2}\left(\frac{2}{N_{f}^{2}}+2\right)\right] \\
\beta_{z_{1}}= & 4 z_{1} a_{H}+z_{1}^{2}\left(\frac{16}{N_{f}^{2}}+4\right)+16 z_{2} z_{1}+12 z_{2}^{2} \\
\beta_{z_{2}}= & -2 x a_{H}^{2}+4 z_{2} a_{H}+\frac{24 z_{1} z_{2}}{N_{f}^{2}}+8 z_{2}^{2} .
\end{aligned}
$$

And for the anomalous dimensions:

$$
\begin{gathered}
\gamma_{H}=a_{H}+2 z_{2}^{2}\left(1+\frac{1}{N_{f}^{2}}\right)-\frac{3 x a_{H}^{2}}{2}+\frac{5 a_{g} a_{H}}{2}\left(1-\frac{x^{2}}{N_{f}^{2}}\right)+2 z_{1}^{2}\left(\frac{1}{N_{f}^{2}}+\frac{1}{N_{f}^{4}}\right)+\frac{8 z_{1} z_{2}}{N_{f}^{2}} \quad \text { (B.5) } \\
\gamma_{S}=\gamma_{\operatorname{Tr}\left[H H^{\dagger}\right]}=2 a_{H}+4 z_{1}\left(1+\frac{1}{N_{f}^{2}}\right)+8 z_{2}-3 a_{H}^{2} x+5 a_{g} a_{H}\left(1-\frac{x^{2}}{N_{f}^{2}}\right)-8 a_{H} z_{1}\left(1+\frac{1}{N_{f}^{2}}\right) \\
-20 z_{1}^{2}\left(\frac{1}{N_{f}^{2}}+\frac{1}{N_{f}^{4}}\right)-16 a_{H} z_{2}-\frac{80}{N_{f}^{2}} z_{1} z_{2}-20 z_{2}^{2}\left(1+\frac{1}{N_{f}^{2}}\right) \\
\gamma_{A}=\gamma_{\operatorname{Tr}\left[T^{a} H T^{a} H^{\dagger}\right]}=2 a_{H}+\frac{4 z_{1}}{N_{f}^{2}}-3 a_{H}^{2} x+5 a_{g} a_{H}\left(1-\frac{x^{2}}{N_{f}^{2}}\right)-\frac{8 a_{H} z_{1}}{N_{f}^{2}} \\
-4 z_{1}^{2}\left(\frac{1}{N_{f}^{2}}+\frac{5}{N_{f}^{4}}\right)-\frac{16 z_{1} z_{2}}{N_{f}^{2}}+4 z_{2}^{2}\left(1-\frac{5}{N_{f}^{2}}\right) .
\end{gathered}
$$

Remarkably all the leading $1 / N_{f}$ corrections emerge only at the order $1 / N_{f}^{2}$ order.

Open Access. This article is distributed under the terms of the Creative Commons Attribution License (CC-BY 4.0), which permits any use, distribution and reproduction in any medium, provided the original author(s) and source are credited.

\section{References}

[1] K.G. Wilson, Renormalization group and critical phenomena. 1. Renormalization group and the Kadanoff scaling picture, Phys. Rev. B 4 (1971) 3174 [InSPIRE].

[2] K.G. Wilson, Renormalization group and critical phenomena. 2. Phase space cell analysis of critical behavior, Phys. Rev. B 4 (1971) 3184 [INSPIRE].

[3] D.J. Gross and F. Wilczek, Ultraviolet Behavior of Nonabelian Gauge Theories, Phys. Rev. Lett. 30 (1973) 1343 [INSPIRE].

[4] H.D. Politzer, Reliable Perturbative Results for Strong Interactions?, Phys. Rev. Lett. 30 (1973) 1346 [InSPIRE]. 
[5] S. Weinberg, Ultraviolet Divergences In Quantum Theories Of Gravitation, in General Relativity: An Einstein centenary survey, S.W. Hawking and W. Istrael eds., Cambridge University Press (1979), pg. 790-831 [INSPIRE].

[6] D.I. Kazakov, Ultraviolet fixed points in gauge and SUSY field theories in extra dimensions, JHEP 03 (2003) 020 [hep-th/0209100] [INSPIRE].

[7] H. Gies, J. Jaeckel and C. Wetterich, Towards a renormalizable standard model without fundamental Higgs scalar, Phys. Rev. D 69 (2004) 105008 [hep-ph/0312034] [INSPIRE].

[8] T.R. Morris, Renormalizable extra-dimensional models, JHEP 01 (2005) 002 [hep-ph/0410142] [INSPIRE].

[9] P. Fischer and D.F. Litim, Fixed points of quantum gravity in extra dimensions, Phys. Lett. B 638 (2006) 497 [hep-th/0602203] [INSPIRE].

[10] P. Fischer and D.F. Litim, Fixed points of quantum gravity in higher dimensions, AIP Conf. Proc. 861 (2006) 336 [hep-th/0606135] [INSPIRE].

[11] D.I. Kazakov and G.S. Vartanov, Renormalizable 1/N(f) Expansion for Field Theories in Extra Dimensions, JHEP 06 (2007) 081 [arXiv:0707.2564] [INSPIRE].

[12] O. Zanusso, L. Zambelli, G.P. Vacca and R. Percacci, Gravitational corrections to Yukawa systems, Phys. Lett. B 689 (2010) 90 [arXiv:0904.0938] [InSPIRE].

[13] H. Gies, S. Rechenberger and M.M. Scherer, Towards an Asymptotic-Safety Scenario for Chiral Yukawa Systems, Eur. Phys. J. C 66 (2010) 403 [arXiv:0907.0327] [InSPIRE].

[14] J.-E. Daum, U. Harst and M. Reuter, Running Gauge Coupling in Asymptotically Safe Quantum Gravity, JHEP 01 (2010) 084 [arXiv:0910.4938] [INSPIRE].

[15] G.P. Vacca and O. Zanusso, Asymptotic Safety in Einstein Gravity and Scalar-Fermion Matter, Phys. Rev. Lett. 105 (2010) 231601 [arXiv: 1009.1735] [INSPIRE].

[16] X. Calmet, Asymptotically safe weak interactions, Mod. Phys. Lett. A 26 (2011) 1571 [arXiv: 1012.5529] [INSPIRE].

[17] S. Folkerts, D.F. Litim and J.M. Pawlowski, Asymptotic freedom of Yang-Mills theory with gravity, Phys. Lett. B 709 (2012) 234 [arXiv:1101.5552] [INSPIRE].

[18] F. Bazzocchi, M. Fabbrichesi, R. Percacci, A. Tonero and L. Vecchi, Fermions and Goldstone bosons in an asymptotically safe model, Phys. Lett. B 705 (2011) 388 [arXiv:1105.1968] [INSPIRE].

[19] H. Gies, S. Rechenberger, M.M. Scherer and L. Zambelli, An asymptotic safety scenario for gauged chiral Higgs-Yukawa models, Eur. Phys. J. C 73 (2013) 2652 [arXiv:1306.6508] [INSPIRE].

[20] O. Antipin, M. Mojaza and F. Sannino, Conformal Extensions of the Standard Model with Veltman Conditions, Phys. Rev. D 89 (2014) 085015 [arXiv:1310.0957] [INSPIRE].

[21] P. Donà, A. Eichhorn and R. Percacci, Matter matters in asymptotically safe quantum gravity, Phys. Rev. D 89 (2014) 084035 [arXiv:1311.2898] [INSPIRE].

[22] A. Bonanno and M. Reuter, Cosmology of the Planck era from a renormalization group for quantum gravity, Phys. Rev. D 65 (2002) 043508 [hep-th/0106133] [INSPIRE].

[23] K.A. Meissner and H. Nicolai, Conformal Symmetry and the Standard Model, Phys. Lett. B 648 (2007) 312 [hep-th/0612165] [INSPIRE]. 
[24] R. Foot, A. Kobakhidze, K. McDonald and R. Volkas, Neutrino mass in radiatively-broken scale-invariant models, Phys. Rev. D 76 (2007) 075014 [arXiv:0706.1829] [InSPIRE].

[25] R. Foot, A. Kobakhidze, K.L. McDonald and R.R. Volkas, A solution to the hierarchy problem from an almost decoupled hidden sector within a classically scale invariant theory, Phys. Rev. D 77 (2008) 035006 [arXiv:0709.2750] [inSPIRE].

[26] J. Hewett and T. Rizzo, Collider Signals of Gravitational Fixed Points, JHEP 12 (2007) 009 [arXiv: 0707.3182] [INSPIRE].

[27] D.F. Litim and T. Plehn, Signatures of gravitational fixed points at the LHC, Phys. Rev. Lett. 100 (2008) 131301 [arXiv: 0707.3983] [INSPIRE].

[28] M. Shaposhnikov and D. Zenhausern, Quantum scale invariance, cosmological constant and hierarchy problem, Phys. Lett. B 671 (2009) 162 [arXiv:0809.3406] [INSPIRE].

[29] M. Shaposhnikov and D. Zenhausern, Scale invariance, unimodular gravity and dark energy, Phys. Lett. B 671 (2009) 187 [arXiv:0809.3395] [InSPIRE].

[30] M. Shaposhnikov and C. Wetterich, Asymptotic safety of gravity and the Higgs boson mass, Phys. Lett. B 683 (2010) 196 [arXiv:0912.0208] [InSPIRE].

[31] S. Weinberg, Asymptotically Safe Inflation, Phys. Rev. D 81 (2010) 083535 [arXiv:0911.3165] [INSPIRE].

[32] G. 't Hooft, Probing the small distance structure of canonical quantum gravity using the conformal group, arXiv: 1009.0669 [INSPIRE].

[33] E. Gerwick, D. Litim and T. Plehn, Asymptotic safety and Kaluza-Klein gravitons at the LHC, Phys. Rev. D 83 (2011) 084048 [arXiv:1101.5548] [INSPIRE].

[34] E. Gerwick, Asymptotically Safe Gravitons in Electroweak Precision Physics, Eur. Phys. J. C 71 (2011) 1676 [arXiv: 1012.1118] [INSPIRE].

[35] M. Hindmarsh, D. Litim and C. Rahmede, Asymptotically Safe Cosmology, JCAP 07 (2011) 019 [arXiv: 1101.5401] [INSPIRE].

[36] T. Hur and P. Ko, Scale invariant extension of the standard model with strongly interacting hidden sector, Phys. Rev. Lett. 106 (2011) 141802 [arXiv:1103.2571] [INSPIRE].

[37] B. Dobrich and A. Eichhorn, Can we see quantum gravity? Photons in the asymptotic-safety scenario, JHEP 06 (2012) 156 [arXiv:1203.6366] [INSPIRE].

[38] G. Marques Tavares, M. Schmaltz and W. Skiba, Higgs mass naturalness and scale invariance in the UV, Phys. Rev. D 89 (2014) 015009 [arXiv:1308.0025] [InSPIRE].

[39] C. Tamarit, Running couplings with a vanishing scale anomaly, JHEP 12 (2013) 098 [arXiv: 1309.0913] [INSPIRE].

[40] S. Abel and A. Mariotti, Novel Higgs Potentials from Gauge Mediation of Exact Scale Breaking, Phys. Rev. D 89 (2014) 125018 [arXiv:1312.5335] [InSPIRE].

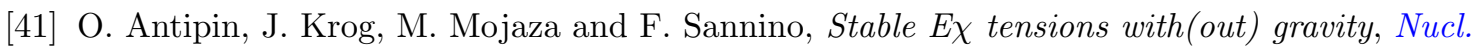
Phys. B 886 (2014) 125 [arXiv:1311.1092] [INSPIRE].

[42] M. Heikinheimo, A. Racioppi, M. Raidal, C. Spethmann and K. Tuominen, Physical Naturalness and Dynamical Breaking of Classical Scale Invariance, Mod. Phys. Lett. A 29 (2014) 1450077 [arXiv: 1304.7006] [INSPIRE]. 
[43] E. Gabrielli, M. Heikinheimo, K. Kannike, A. Racioppi, M. Raidal and C. Spethmann, Towards Completing the Standard Model: Vacuum Stability, EWSB and Dark Matter, Phys. Rev. D 89 (2014) 015017 [arXiv: 1309.6632] [INSPIRE].

[44] M. Holthausen, J. Kubo, K.S. Lim and M. Lindner, Electroweak and Conformal Symmetry Breaking by a Strongly Coupled Hidden Sector, JHEP 12 (2013) 076 [arXiv:1310.4423] [INSPIRE].

[45] G.C. Dorsch, S.J. Huber and J.M. No, Cosmological Signatures of a UV-Conformal Standard Model, Phys. Rev. Lett. 113 (2014) 121801 [arXiv:1403.5583] [INSPIRE].

[46] A. Eichhorn and M.M. Scherer, Planck scale, Higgs mass and scalar dark matter, Phys. Rev. D 90 (2014) 025023 [arXiv: 1404.5962] [INSPIRE].

[47] D.F. Litim, Renormalisation group and the Planck scale, Phil. Trans. Roy. Soc. Lond. A 369 (2011) 2759 [arXiv: 1102 .4624] [inSPIRE].

[48] D.F. Litim, On fixed points of quantum gravity, AIP Conf. Proc. 841 (2006) 322 [hep-th/0606044] [INSPIRE].

[49] M. Niedermaier, The asymptotic safety scenario in quantum gravity: An introduction, Class. Quant. Grav. 24 (2007) R171 [gr-qc/0610018] [INSPIRE].

[50] M. Niedermaier and M. Reuter, The Asymptotic Safety Scenario in Quantum Gravity, Living Rev. Rel. 9 (2006) 5.

[51] R. Percacci, Asymptotic Safety, arXiv:0709.3851 [INSPIRE].

[52] D.F. Litim, Fixed Points of Quantum Gravity and the Renormalisation Group, PoS (QG-Ph) 024 [arXiv:0810.3675] [INSPIRE].

[53] M. Reuter and F. Saueressig, Quantum Einstein Gravity, New J. Phys. 14 (2012) 055022 [arXiv: 1202.2274] [INSPIRE].

[54] D.F. Litim and F. Sannino, Asymptotic safety guaranteed, JHEP 12 (2014) 178 [arXiv: 1406.2337] [INSPIRE].

[55] F. Sannino, Conformal Dynamics for TeV Physics and Cosmology, Acta Phys. Polon. B 40 (2009) 3533 [arXiv: 0911.0931] [INSPIRE].

[56] T. Appelquist and F. Sannino, The physical spectrum of conformal $\mathrm{SU}(N)$ gauge theories, Phys. Rev. D 59 (1999) 067702 [hep-ph/9806409] [INSPIRE].

[57] J.T. Lenaghan, F. Sannino and K. Splittorff, The superfluid and conformal phase transitions of two color QCD, Phys. Rev. D 65 (2002) 054002 [hep-ph/0107099] [INSPIRE].

[58] D.D. Dietrich and F. Sannino, Conformal window of $\mathrm{SU}(N)$ gauge theories with fermions in higher dimensional representations, Phys. Rev. D 75 (2007) 085018 [hep-ph/0611341] [INSPIRE].

[59] T.A. Ryttov and F. Sannino, Conformal Windows of $\mathrm{SU}(N)$ Gauge Theories, Higher Dimensional Representations and The Size of The Unparticle World, Phys. Rev. D 76 (2007) 105004 [arXiv:0707.3166] [rNSPIRE].

[60] F. Sannino, Conformal Chiral Dynamics, Phys. Rev. D 80 (2009) 017901 [arXiv:0811.0616] [INSPIRE].

[61] F. Sannino, Conformal Windows of $\mathrm{Sp}(2 N)$ and $\mathrm{SO}(N)$ Gauge Theories, Phys. Rev. D 79 (2009) 096007 [arXiv:0902.3494] [INSPIRE]. 
[62] M. Jarvinen and F. Sannino, Holographic Conformal Window - A Bottom Up Approach, JHEP 05 (2010) 041 [arXiv:0911.2462] [INSPIRE].

[63] O. Antipin and K. Tuominen, Constraints on Conformal Windows from Holographic Duals, Mod. Phys. Lett. A 26 (2011) 2227 [arXiv:0912.0674] [INSPIRE].

[64] H.S. Fukano and F. Sannino, Conformal Window of Gauge Theories with Four-Fermion Interactions and Ideal Walking, Phys. Rev. D 82 (2010) 035021 [arXiv:1005.3340] [INSPIRE].

[65] T.A. Ryttov and R. Shrock, Infrared Evolution and Phase Structure of a Gauge Theory Containing Different Fermion Representations, Phys. Rev. D 81 (2010) 116003 [arXiv:1006.0421] [INSPIRE].

[66] T.A. Ryttov and R. Shrock, Higher-Loop Corrections to the Infrared Evolution of a Gauge Theory with Fermions, Phys. Rev. D 83 (2011) 056011 [arXiv:1011.4542] [INSPIRE].

[67] F. Sannino, Mass Deformed Exact S-parameter in Conformal Theories, Phys. Rev. D 82 (2010) 081701 [arXiv: 1006.0207] [INSPIRE].

[68] M. Mojaza, C. Pica and F. Sannino, Hot Conformal Gauge Theories, Phys. Rev. D 82 (2010) 116009 [arXiv:1010.4798] [INSPIRE].

[69] C. Pica and F. Sannino, $\beta$-function and Anomalous Dimensions, Phys. Rev. D 83 (2011) 116001 [arXiv: 1011.3832] [INSPIRE].

[70] C. Pica and F. Sannino, UV and IR Zeros of Gauge Theories at The Four Loop Order and Beyond, Phys. Rev. D 83 (2011) 035013 [arXiv:1011.5917] [inSPIRE].

[71] O. Antipin, M. Mojaza, C. Pica and F. Sannino, Magnetic Fixed Points and Emergent Supersymmetry, JHEP 06 (2013) 037 [arXiv:1105.1510] [INSPIRE].

[72] T.A. Ryttov and R. Shrock, Scheme Transformations in the Vicinity of an Infrared Fixed Point, Phys. Rev. D 86 (2012) 065032 [arXiv:1206.2366] [InSPIRE].

[73] M. Mojaza, C. Pica, T.A. Ryttov and F. Sannino, Exceptional and Spinorial Conformal Windows, Phys. Rev. D 86 (2012) 076012 [arXiv:1206.2652] [INSPIRE].

[74] R. Foadi and F. Sannino, $S$ and $T$ parameters from a light nonstandard Higgs particle, Phys. Rev. D 87 (2013) 015008 [arXiv:1207.1541] [INSPIRE].

[75] O. Antipin, M. Mojaza and F. Sannino, Jumping out of the light-Higgs conformal window, Phys. Rev. D 87 (2013) 096005 [arXiv: 1208.0987] [inSPIRE].

[76] T.A. Ryttov and R. Shrock, An Analysis of Scheme Transformations in the Vicinity of an Infrared Fixed Point, Phys. Rev. D 86 (2012) 085005 [arXiv:1206.6895] [INSPIRE].

[77] T.A. Ryttov, Higher loop corrections to the infrared evolution of fermionic gauge theories in the RI'scheme, Phys. Rev. D 89 (2014) 016013 [arXiv:1309.3867] [INSPIRE].

[78] O. Antipin, M. Gillioz and F. Sannino, a New Conformal Window Bound from the a theorem, arXiv: 1303.1547 [INSPIRE].

[79] T.A. Ryttov, Infrared fixed points in the minimal momentum subtraction scheme, Phys. Rev. D 89 (2014) 056001 [arXiv:1311.0848] [INSPIRE].

[80] E. Mølgaard and R. Shrock, Renormalization-Group Flows and Fixed Points in Yukawa Theories, Phys. Rev. D 89 (2014) 105007 [arXiv:1403.3058] [INSPIRE]. 
[81] R. Shrock, Generalized Scheme Transformations for the Elimination of Higher-Loop Terms in the $\beta$-function of a Gauge Theory, Phys. Rev. D 90 (2014) 045011 [arXiv:1405.6244] [INSPIRE].

[82] S. Catterall and F. Sannino, Minimal walking on the lattice, Phys. Rev. D 76 (2007) 034504 [arXiv:0705.1664] [INSPIRE].

[83] S. Catterall, J. Giedt, F. Sannino and J. Schneible, Phase diagram of SU(2) with 2 flavors of dynamical adjoint quarks, JHEP 11 (2008) 009 [arXiv:0807.0792] [INSPIRE].

[84] L. Del Debbio, A. Patella and C. Pica, Fermions in higher representations. Some results about SU(2) with adjoint fermions, PoS(LATTICE 2008) 064 [arXiv:0812.0570] [INSPIRE].

[85] L. Del Debbio, B. Lucini, A. Patella, C. Pica and A. Rago, Conformal versus confining scenario in $\mathrm{SU}(2)$ with adjoint fermions, Phys. Rev. D 80 (2009) 074507 [arXiv:0907.3896] [INSPIRE].

[86] A. Deuzeman, M.P. Lombardo and E. Pallante, Evidence for a conformal phase in $\mathrm{SU}(N)$ gauge theories, Phys. Rev. D 82 (2010) 074503 [arXiv:0904.4662] [INSPIRE].

[87] L. Del Debbio, B. Lucini, A. Patella, C. Pica and A. Rago, The infrared dynamics of Minimal Walking Technicolor, Phys. Rev. D 82 (2010) 014510 [arXiv:1004.3206] [InSPIRE].

[88] L. Del Debbio, B. Lucini, A. Patella, C. Pica and A. Rago, Mesonic spectroscopy of Minimal Walking Technicolor, Phys. Rev. D 82 (2010) 014509 [arXiv:1004.3197] [InSPIRE].

[89] Z. Fodor, K. Holland, J. Kuti, D. Nogradi and C. Schroeder, Nearly conformal gauge theories in finite volume, Phys. Lett. B 681 (2009) 353 [arXiv:0907.4562] [INSPIRE].

[90] Z. Fodor, K. Holland, J. Kuti, D. Nogradi and C. Schroeder, Chiral properties of SU(3) sextet fermions, JHEP 11 (2009) 103 [arXiv:0908.2466] [INSPIRE].

[91] Z. Fodor, K. Holland, J. Kuti, D. Nogradi and C. Schroeder, Chiral symmetry breaking in nearly conformal gauge theories, PoS(LAT2009) 055 [arXiv:0911.2463] [INSPIRE].

[92] F. Bursa et al., Improved Lattice Spectroscopy of Minimal Walking Technicolor, Phys. Rev. D 84 (2011) 034506 [arXiv: 1104.4301] [INSPIRE].

[93] A.J. Hietanen, J. Rantaharju, K. Rummukainen and K. Tuominen, Spectrum of SU(2) lattice gauge theory with two adjoint Dirac flavours, JHEP 05 (2009) 025 [arXiv:0812.1467] [INSPIRE].

[94] T. Appelquist, G.T. Fleming, M.F. Lin, E.T. Neil and D.A. Schaich, Lattice Simulations and Infrared Conformality, Phys. Rev. D 84 (2011) 054501 [arXiv:1106.2148] [INSPIRE].

[95] A.J. Hietanen, K. Rummukainen and K. Tuominen, Evolution of the coupling constant in $\mathrm{SU}(2)$ lattice gauge theory with two adjoint fermions, Phys. Rev. D 80 (2009) 094504 [arXiv: 0904.0864] [INSPIRE].

[96] A. Hietanen, J. Rantaharju, K. Rummukainen and K. Tuominen, Minimal technicolor on the lattice, Nucl. Phys. A 820 (2009) 191C [inSPIRE].

[97] F. Bursa, L. Del Debbio, L. Keegan, C. Pica and T. Pickup, Running of the coupling and quark mass in $\mathrm{SU}(2)$ with two adjoint fermions, PoS (LAT2009) 056 [arXiv:0910.2562] [INSPIRE].

[98] F. Bursa, L. Del Debbio, L. Keegan, C. Pica and T. Pickup, Mass anomalous dimension in SU(2) with two adjoint fermions, Phys. Rev. D 81 (2010) 014505 [arXiv:0910.4535] [INSPIRE]. 
[99] L. Del Debbio, B. Lucini, C. Pica, A. Patella, A. Rago and S. Roman, Large-volume results in $\mathrm{SU}(2)$ with adjoint fermions, arXiv: 1311.5597 [INSPIRE].

[100] T. Banks and A. Zaks, On the Phase Structure of Vector-Like Gauge Theories with Massless Fermions, Nucl. Phys. B 196 (1982) 189 [InSPIRE].

[101] O. Antipin, M. Mojaza and F. Sannino, Light Dilaton at Fixed Points and Ultra Light Scale Super Yang-Mills, Phys. Lett. B 712 (2012) 119 [arXiv:1107.2932] [INSPIRE].

[102] O. Antipin, S. Di Chiara, M. Mojaza, E. Mølgaard and F. Sannino, A Perturbative Realization of Miransky Scaling, Phys. Rev. D 86 (2012) 085009 [arXiv:1205.6157] [INSPIRE].

[103] O. Antipin, M. Gillioz, E. Mølgaard and F. Sannino, The a theorem for gauge-Yukawa theories beyond Banks-Zaks fixed point, Phys. Rev. D 87 (2013) 125017 [arXiv:1303.1525] [INSPIRE].

[104] O. Antipin, M. Gillioz, J. Krog, E. Mølgaard and F. Sannino, Standard Model Vacuum Stability and Weyl Consistency Conditions, JHEP 08 (2013) 034 [arXiv:1306.3234] [INSPIRE].

[105] R. Rattazzi, V.S. Rychkov, E. Tonni and A. Vichi, Bounding scalar operator dimensions in 4D CFT, JHEP 12 (2008) 031 [arXiv:0807.0004] [inSPIRE].

[106] V.S. Rychkov and A. Vichi, Universal Constraints on Conformal Operator Dimensions, Phys. Rev. D 80 (2009) 045006 [arXiv:0905.2211] [INSPIRE].

[107] D. Poland, D. Simmons-Duffin and A. Vichi, Carving Out the Space of 4 D CFTs, JHEP 05 (2012) 110 [arXiv:1109.5176] [INSPIRE].

[108] G. Mack, All Unitary Ray Representations of the Conformal Group SU(2,2) with Positive Energy, Commun. Math. Phys. 55 (1977) 1 [inSPIRE].

[109] S. El-Showk and M.F. Paulos, Bootstrapping Conformal Field Theories with the Extremal Functional Method, Phys. Rev. Lett. 111 (2013) 241601 [arXiv:1211.2810] [INSPIRE].

[110] Y. Nakayama and T. Ohtsuki, Approaching the conformal window of $O(n) \times O(m)$ symmetric Landau-Ginzburg models using the conformal bootstrap, Phys. Rev. D 89 (2014) 126009 [arXiv: 1404.0489] [INSPIRE].

[111] P. Di Francesco, P. Mathieu and D. Senechal, Conformal Field Theory, Springer, New York, U.S.A. (1997), pg. 890 [inSPIRE].

[112] F.A. Dolan and H. Osborn, Conformal four point functions and the operator product expansion, Nucl. Phys. B 599 (2001) 459 [hep-th/0011040] [INSPIRE].

[113] F.A. Dolan and H. Osborn, Conformal partial waves and the operator product expansion, Nucl. Phys. B 678 (2004) 491 [hep-th/0309180] [INSPIRE].

[114] R. Rattazzi, S. Rychkov and A. Vichi, Bounds in 4D Conformal Field Theories with Global Symmetry, J. Phys. A 44 (2011) 035402 [arXiv:1009.5985] [InSPIRE].

[115] M.-x. Luo, H.-w. Wang and Y. Xiao, Two loop renormalization group equations in general gauge field theories, Phys. Rev. D 67 (2003) 065019 [hep-ph/0211440] [INSPIRE].

[116] M.E. Machacek and M.T. Vaughn, Two Loop Renormalization Group Equations in a General Quantum Field Theory. 1. Wave Function Renormalization, Nucl. Phys. B 222 (1983) 83 [INSPIRE]. 
[117] M.E. Machacek and M.T. Vaughn, Two Loop Renormalization Group Equations in a General Quantum Field Theory. 2. Yukawa Couplings, Nucl. Phys. B 236 (1984) 221 [INSPIRE].

[118] M.E. Machacek and M.T. Vaughn, Two Loop Renormalization Group Equations in a General Quantum Field Theory. 3. Scalar Quartic Couplings, Nucl. Phys. B 249 (1985) 70 [INSPIRE].

[119] H. Osborn, Weyl consistency conditions and a local renormalization group equation for general renormalizable field theories, Nucl. Phys. B 363 (1991) 486 [InSPIRE].

[120] E. Mølgaard, Decrypting gauge-Yukawa cookbooks, Eur. Phys. J. Plus 129 (2014) 159 [arXiv: 1404.5550] [INSPIRE].

[121] I. Heemskerk, J. Penedones, J. Polchinski and J. Sully, Holography from Conformal Field Theory, JHEP 10 (2009) 079 [arXiv:0907.0151] [INSPIRE].

[122] Y. Makeenko, Large-N gauge theories, NATO Sci. Ser. C 556 (2000) 285 [hep-th/0001047] [INSPIRE].

[123] N. Seiberg, Electric-magnetic duality in supersymmetric nonAbelian gauge theories, Nucl. Phys. B 435 (1995) 129 [hep-th/9411149] [INSPIRE].

[124] F. Sannino, QCD Dual, Phys. Rev. D 80 (2009) 065011 [arXiv:0907.1364] [InSPIRE].

[125] F. Sannino, Magnetic S-parameter, Phys. Rev. Lett. 105 (2010) 232002 [arXiv:1007.0254] [INSPIRE]. 